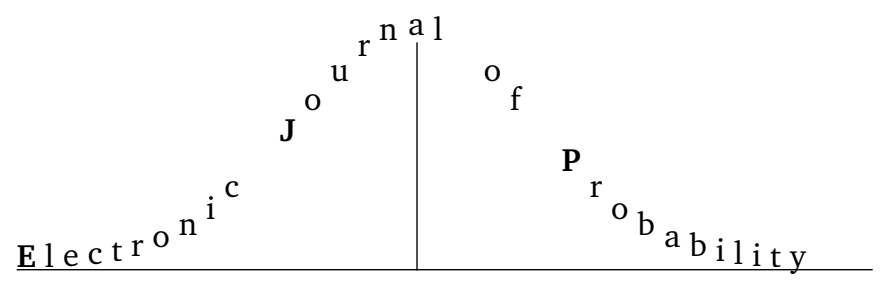

Vol. 14 (2009), Paper no. 5, pages 87-118.

Journal URL

http://www.math.washington.edu/ ejpecp/

\title{
On percolation in random graphs with given vertex degrees
}

\author{
Svante Janson \\ Department of Mathematics \\ Uppsala University \\ PO Box 480 \\ SE-751 06 Uppsala \\ Sweden \\ svante.janson@math.uu.se \\ http://www.math.uu.se/ svante/
}

\begin{abstract}
We study the random graph obtained by random deletion of vertices or edges from a random graph with given vertex degrees. A simple trick of exploding vertices instead of deleting them, enables us to derive results from known results for random graphs with given vertex degrees. This is used to study existence of giant component and existence of $k$-core. As a variation of the latter, we study also bootstrap percolation in random regular graphs.

We obtain both simple new proofs of known results and new results. An interesting feature is that for some degree sequences, there are several or even infinitely many phase transitions for the $k$-core.
\end{abstract}

Key words: random graph, giant component, k-core, bootstrap percolation.

AMS 2000 Subject Classification: Primary 60C05; 05C80.

Submitted to EJP on April 10, 2008, final version accepted December 17, 2008. 


\section{Introduction}

One popular and important type of random graph is given by the uniformly distributed random graph with a given degree sequence, defined as follows. Let $n \in \mathbb{N}$ and let $\mathbf{d}=\left(d_{i}\right)_{1}^{n}$ be a sequence of non-negative integers. We let $G(n, \mathbf{d})$ be a random graph with degree sequence $\mathbf{d}$, uniformly chosen among all possibilities (tacitly assuming that there is any such graph at all; in particular, $\sum_{i} d_{i}$ has to be even).

It is well-known that it is often simpler to study the corresponding random multigraph $G^{*}(n, \mathbf{d})$ with given degree sequence $\mathbf{d}=\left(d_{i}\right)_{1}^{n}$, defined for every sequence $\mathbf{d}$ with $\sum_{i} d_{i}$ even by the configuration model (see e.g. Bollobás [3]): take a set of $d_{i}$ half-edges for each vertex $i$, and combine the halfedges into pairs by a uniformly random matching of the set of all half-edges (this pairing is called a configuration); each pair of half-edges is then joined to form an edge of $G^{*}(n, \mathbf{d})$.

We consider asymptotics as the numbers of vertices tend to infinity, and thus we assume throughout the paper that we are given, for each $n$, a sequence $\mathbf{d}^{(n)}=\left(d_{i}^{(n)}\right)_{1}^{n}$ with $\sum_{i} d_{i}^{(n)}$ even. (As usual, we could somewhat more generally assume that we are given a sequence $n_{v} \rightarrow \infty$ and for each $v$ a sequence $\mathbf{d}^{(v)}=\left(d_{i}^{(v)}\right)_{1}^{n_{v}}$.) For notational simplicity we will usually not show the dependency on $n$ explicitly; we thus write $\mathbf{d}$ and $d_{i}$, and similarly for other (deterministic or random) quantities introduced below. All unspecified limits and other asymptotic statements are for $n \rightarrow \infty$. For example, w.h.p. (with high probability) means 'with probability tending to 1 as $n \rightarrow \infty$ ', and $\stackrel{\mathrm{p}}{\longrightarrow}$ means 'convergence in probability as $n \rightarrow \infty$ '. Similarly, we use $o_{p}$ and $O_{p}$ in the standard way, always implying $n \rightarrow \infty$. For example, if $X$ is a parameter of the random graph, $X=o_{p}(n)$ means that $\mathbb{P}(X>\varepsilon n) \rightarrow 0$ as $n \rightarrow \infty$ for every $\varepsilon>0$; equivalently, $X / n \stackrel{\mathrm{p}}{\longrightarrow} 0$.

We may obtain $G(n, \mathbf{d})$ by conditioning the multigraph $G^{*}(n, \mathbf{d})$ on being a (simple) graph, i.e., on not having any multiple edges or loops. By Janson [9] (with earlier partial results by many authors),

$$
\liminf \mathbb{P}\left(G^{*}(n, \mathbf{d}) \text { is simple }\right)>0 \Longleftrightarrow \sum_{i=1}^{n} d_{i}^{2}=O\left(\sum_{i=1}^{n} d_{i}\right) .
$$

In this case, many results transfer immediately from $G^{*}(n, \mathbf{d})$ to $G(n, \mathbf{d})$, for example, every result of the type $\mathbb{P}\left(\mathscr{E}_{n}\right) \rightarrow 0$ for some events $\mathscr{E}_{n}$, and thus every result saying that some parameter converges in probability to some non-random value. This includes every result in the present paper.

We will in this paper study the random multigraph $G^{*}(n, \mathbf{d})$; the reader can think of doing this either for its own sake or as a tool for studying $G(n, \mathbf{d})$. We leave the statement of corollaries for $G(n, \mathbf{d})$, using (1.1), to the reader. Moreover, the results for $G(n, \mathbf{d})$ extend to some other random graph models too, in particular $G(n, p)$ with $p \sim \lambda / n$ and $G(n, m)$ with $m \sim \lambda n / 2$ with $\lambda>0$, by the standard device of conditioning on the degree sequence; again we omit the details and refer to $[10 ; 11 ; 12]$ where this method is used.

We will consider percolation of these random (multi)graphs, where we first generate a random graph $G^{*}(n, \mathbf{d})$ and then delete either vertices or edges at random. (From now on, we simply write 'graph' for 'multigraph'.) The methods below can be combined to treat the case of random deletion of both vertices and edges, which is studied by other methods in e.g. Britton, Janson and Martin-Löf [4], but we leave this to the reader.

To be precise, we consider the following two constructions, given any graph $G$ and a probability $\pi \in[0,1]$. 
Site percolation Randomly delete each vertex (together with all incident edges) with probability $1-\pi$, independently of all other vertices. We denote the resulting random graph by $G_{\pi, \mathrm{v}}$.

Bond percolation Randomly delete each edge with probability $1-\pi$, independently of all other edges. (All vertices are left.) We denote the resulting random graph by $G_{\pi, \mathrm{e}}$.

Thus $\pi$ denotes the probability to be kept in the percolation model. When, as in our case, the original graph $G$ itself is random, it is further assumed that we first sample $G$ and then proceed as above, conditionally on $G$.

The cases $\pi=0,1$ are trivial: $G_{1, \mathrm{v}}=G_{1, \mathrm{e}}=G$, while $G_{0, \mathrm{v}}=\emptyset$, the null graph with no vertices and no edges, and $G_{0, \mathrm{e}}$ is the empty graph with the same vertex set as $G$ but no edges. We will thus mainly consider $0<\pi<1$.

We may generalize the site percolation model by letting the probability depend on the degree of the vertex. Thus, if $\pi=\left(\pi_{d}\right)_{0}^{\infty}$ is a given sequence of probabilities $\pi_{d} \in[0,1]$, let $G_{\pi, \mathrm{v}}$ be the random graph obtained by deleting vertices independently of each other, with vertex $v \in G$ deleted with probability $1-\pi_{d(v)}$ where $d(v)$ is the degree of $v$ in $G$.

For simplicity and in order to concentrate on the main ideas, we will in this paper consider only the case when the probability $\pi$ (or the sequence $\pi$ ) is fixed and thus does not depend on $n$, with the exception of a few remarks where we briefly indicate how the method can be used also for a more detailed study of thresholds.

The present paper is inspired by Fountoulakis [7], and we follow his idea of deriving results for the percolation models $G^{*}(n, \mathbf{d})_{\pi, \mathrm{v}}$ and $G^{*}(n, \mathbf{d})_{\pi, \mathrm{e}}$ from results for the model $G^{*}(n, \mathbf{d})$ without deletions, but for different degree sequences $\mathbf{d}$. We will, however, use another method to do this, which we find simpler.

Fountoulakis [7] shows that for both site and bond percolation on $G^{*}(n, \mathbf{d})$, if we condition the resulting random graph on its degree sequence $\mathbf{d}^{\prime}$, and let $n^{\prime}$ be the number of its vertices, then the graph has the distribution of $G^{*}\left(n^{\prime}, \mathbf{d}^{\prime}\right)$, the random graph with this degree sequence constructed by the configuration model. He then proceeds to calculate the distributions of the degree sequence $\mathbf{d}^{\prime}$ for the two percolation models and finally applies known results to $G^{*}\left(n^{\prime}, \mathbf{d}^{\prime}\right)$.

Our method is a version of this, where we do the deletions in two steps. For site percolation, instead of deleting a vertex, let us first explode it by replacing it by $d$ new vertices of degree 1 , where $d$ is its degree; we further colour the new vertices red. Then clean up by removing all red vertices. Note that the (random) explosions change the number of vertices, but not the number of half-edges. Moreover, given the set of explosions, there is a one-to-one correspondence between configurations before and after the explosions, and thus, if we condition on the new degree sequence, the exploded graph is still described by the configuration model. Furthermore, by symmetry, when removing the red vertices, all vertices of degree 1 are equivalent, so we may just as well remove the right number of vertices of degree 1 , but choose them uniformly at random. Hence, we can obtain $G^{*}(n, \mathbf{d})_{\pi, v}$ as follows:

Site percolation For each vertex $i$, replace it with probability $1-\pi$ by $d_{i}$ new vertices of degree 1 (independently of all other vertices). Let $\tilde{\mathbf{d}}_{\pi, v}$ be the resulting (random) degree sequence, let $\tilde{n}$ be its length (the number of vertices), and let $n_{+}$be the number of new vertices. Construct the random graph $G^{*}\left(\tilde{n}, \tilde{\mathbf{d}}_{\pi, v}\right)$. Finish by deleting $n_{+}$randomly chosen vertices of degree 1 .

The more general case when we are given a sequence $\pi=\left(\pi_{d}\right)_{0}^{\infty}$ is handled in the same way: 
Site percolation, general For each vertex $i$, replace it with probability $1-\pi_{d_{i}}$ by $d_{i}$ new vertices of degree 1. Let $\tilde{\mathbf{d}}_{\pi, v}$ be the resulting (random) degree sequence, let $\tilde{n}$ be its length (the number of vertices), and let $n_{+}$be the number of new vertices. Construct the random graph $G^{*}\left(\tilde{n}, \tilde{\mathbf{d}}_{\pi, \mathrm{v}}\right)$. Finish by deleting $n_{+}$randomly chosen vertices of degree 1 .

Remark 1.1. We have here assumed that vertices are deleted at random, independently of each other. This is not essential for our method, which may be further extended to the case when we remove a set of vertices determined by any random procedure that is independent of the edges in $G(n, \mathbf{d})$ (but may depend on the vertex degrees). For example, we may remove a fixed number $m$ of vertices, chosen uniformly at random. It is easily seen that if $m / n \rightarrow \pi$, the results of Subsection 2.1 below still hold (with all $\pi_{j}=\pi$ ), and thus the results of the later sections hold too. Another, deterministic, example is to remove the first $m$ vertices.

For bond percolation, we instead explode each half-edge with probability $1-\sqrt{\pi}$, independently of all other half-edges; to explode a half-edge means that we disconnect it from its vertex and transfer it to a new, red vertex of degree 1. Again this does not change the number of half-edges, and there is a one-to-one correspondence between configurations before and after the explosions. We finish by removing all red vertices and their incident edges. Since an edge consists of two half-edges, and each survives with probability $\sqrt{\pi}$, this gives the bond percolation model $G^{*}(n, \mathbf{d})_{\pi, \mathrm{e}}$ where edges are kept with probability $\pi$. This yields the following recipe:

Bond percolation Replace the degrees $d_{i}$ in the sequence $\mathbf{d}$ by independent random degrees $\tilde{d}_{i} \sim$ $\operatorname{Bi}\left(d_{i}, \sqrt{\pi}\right)$. (I.e., $\tilde{d}_{i}$ has the binomial distribution $\operatorname{Bi}\left(d_{i}, \sqrt{\pi}\right)$.) Add $n_{+}:=\sum_{i=1}^{n}\left(d_{i}-\tilde{d}_{i}\right)$ new degrees 1 to the sequence $\left(\tilde{d}_{i}\right)_{1}^{n}$, and let $\tilde{\mathbf{d}}_{\pi, \mathrm{e}}$ be the resulting degree sequence and $\tilde{n}=n+n_{+}$ its length. Construct the random graph $G^{*}\left(\tilde{n}, \tilde{\mathbf{d}}_{\pi, \mathrm{e}}\right)$. Finish by deleting $n_{+}$randomly chosen vertices of degree 1 .

In both cases, we have reduced the problem to a simple (random) modification of the degree sequence, plus a random removal of a set of vertices of degree 1. The latter is often more or less trivial to handle, see the applications below. We continue to call the removed vertices red when convenient.

Of course, to use this method, it is essential to find the degree sequence $\tilde{\mathbf{d}}$ after the explosions. We study this in Section 2. We then apply this method to three different problems:

Existence of a giant component in the percolated graph, i.e., what is called percolation in random graph theory (Section 3). Our results include and extend earlier work by Fountoulakis [7], which inspired the present study, and some of the results by Britton, Janson and Martin-Löf [4].

Existence of a $k$-core in the percolated graph (Section 4). We obtain a general result analogous to (and extending) the well-known result by Pittel, Spencer and Wormald [17] for $G(n, p)$. We study the phase transitions that may occur in some detail and show by examples that it is possible to have several, and even an infinite number of, different phase transitions as the probability $\pi$ increases from 0 to 1 .

Bootstrap percolation in random regular graphs (Section 5), where we obtain a new and simpler proof of results by Balogh and Pittel [1].

For a graph $G$, let $v(G)$ and $e(G)$ denote the numbers of vertices and edges in $G$, respectively, and let $v_{j}(G)$ be the number of vertices of degree $j, j \geq 0$. We sometimes use $G^{*}(n, \mathbf{d})_{\pi}$ to denote any of the percolation models $G^{*}(n, \mathbf{d})_{\pi, \mathrm{v}}, G^{*}(n, \mathbf{d})_{\pi, \mathrm{v}}$ or $G^{*}(n, \mathbf{d})_{\pi, \mathrm{e}}$. 


\section{The degree sequence after explosions}

Let $n_{j}:=\#\left\{i \leq n: d_{i}=j\right\}$. Thus $\sum_{j=0}^{\infty} n_{j}=n$, and $n_{j}$ equals the number $v_{j}\left(G^{*}(n, \mathbf{d})\right)$ of vertices of degree $j$ in $G^{*}(n, \mathbf{d})$. We assume for simplicity the following regularity condition.

Condition 2.1. There exists a probability distribution $\left(p_{j}\right)_{j=0}^{\infty}$ with finite positive mean $\lambda:=$ $\sum_{j} j p_{j} \in(0, \infty)$ such that $($ as $n \rightarrow \infty)$

$$
n_{j} / n \rightarrow p_{j}, \quad j \geq 0
$$

and

$$
\frac{\sum_{j=0}^{\infty} j n_{j}}{n} \rightarrow \lambda:=\sum_{j=0}^{\infty} j p_{j} .
$$

Note that, in order to avoid trivialities, we assume that $\lambda>0$, which is equivalent to $p_{0}<1$. Thus, there is a positive fraction of vertices of degree at least 1 .

Note that $\sum_{j} j n_{j}=\sum_{i} d_{i}$ equals twice the number of edges in $G^{*}(n, \mathbf{d})$, and that (2.2) says that the average degree in $G^{*}(n, \mathbf{d})$ converges to $\lambda$.

Let the random variable $\hat{D}=\hat{D}_{n}$ be the degree of a random vertex in $G^{*}(n, \mathbf{d})$, thus $\hat{D}_{n}$ has the distribution $\mathbb{P}\left(\hat{D}_{n}=j\right)=n_{j} / n$, and let $D$ be a random variable with the distribution $\left(p_{j}\right)_{0}^{\infty}$. Then (2.1) is equivalent to $\hat{D}_{n} \stackrel{\mathrm{d}}{\longrightarrow} D$, and (2.2) is $\mathbb{E} \hat{D}_{n} \rightarrow \lambda=\mathbb{E} D$. Further, assuming (2.1), (2.2) is equivalent to uniform integrability of $\hat{D}_{n}$, or equivalently uniform summability (as $n \rightarrow \infty$ ) of $\sum_{j} j n_{j} / n$, see for example Gut [8], Theorem 5.5.9 and Remark 5.5.4.

Remark 2.2. The uniform summability of $\sum_{j} j n_{j} / n$ is easily seen to imply that if $H$ is any (random or deterministic) subgraph on $G^{*}(n, \mathbf{d})$ with $v(H)=o(n)$, then $e(H)=o(n)$, and similarly with $o_{p}(n)$.

We will also use the probability generating function of the asymptotic degree distribution $D$ :

$$
g_{D}(x):=\mathbb{E} x^{D}=\sum_{j=0}^{\infty} p_{j} x^{j},
$$

defined at least for $|x| \leq 1$.

We perform either site or bond percolation as in Section 1 , by the explosion method described there, and let $\tilde{n}_{j}:=\#\left\{i \leq \tilde{n}: \tilde{d}_{i}=j\right\}$ be the number of vertices of degree $j$ after the explosions. Thus

$$
\sum_{j=0}^{\infty} \tilde{n}_{j}=\tilde{n} .
$$

It is easy to find the distribution of $\left(\tilde{n}_{j}\right)$ and its asymptotics for our two percolation models. 


\subsection{Site percolation}

We treat the general version with a sequence $\pi$. Let $n_{j}^{\circ}$ be the number of vertices of degree $j$ that are not exploded. Then

$$
\begin{aligned}
& n_{j}^{\circ} \sim \operatorname{Bi}\left(n_{j}, \pi_{j}\right) \quad \text { (independent of each other), } \\
& n_{+}=\sum_{j=0}^{\infty} j\left(n_{j}-n_{j}^{\circ}\right) \\
& \tilde{n}_{j}=n_{j}^{\circ}, \quad j \neq 1 \\
& \tilde{n}_{1}=n_{1}^{\circ}+n_{+} \cdot
\end{aligned}
$$

By the law of large numbers, $n_{j}^{\circ}=n_{j} \pi_{j}+o_{p}(n)$ and thus, using the assumption (2.1) and the uniform summability of $\sum_{j} j n_{j} / n$ (which enables us to treat the infinite sums in (2.10) and (2.13) by a standard argument),

$$
\begin{aligned}
n_{j}^{\circ} & =n_{j} \pi_{j}+o_{p}(n)=\pi_{j} p_{j} n+o_{p}(n), \\
n_{+} & =\sum_{j=0}^{\infty} j\left(1-\pi_{j}\right) p_{j} n+o_{p}(n), \\
\tilde{n}_{j} & =\pi_{j} p_{j} n+o_{p}(n), \quad j \neq 1, \\
\tilde{n}_{1} & =\left(\pi_{1} p_{1}+\sum_{j=0}^{\infty} j\left(1-\pi_{j}\right) p_{j}\right) n+o_{p}(n), \\
\tilde{n} & =\sum_{j=0}^{\infty}\left(\pi_{j}+j\left(1-\pi_{j}\right)\right) p_{j} n+o_{p}(n) .
\end{aligned}
$$

We can write $(2.13)$ as

$$
\frac{\tilde{n}}{n} \stackrel{\mathrm{p}}{\longrightarrow} \zeta:=\sum_{j=0}^{\infty}\left(\pi_{j}+j\left(1-\pi_{j}\right)\right) p_{j}>0 .
$$

Further, by (2.11) and (2.12),

$$
\frac{\tilde{n}_{j}}{\tilde{n}} \stackrel{\mathrm{p}}{\longrightarrow} \tilde{p}_{j}:= \begin{cases}\zeta^{-1} \pi_{j} p_{j}, & j \neq 1, \\ \zeta^{-1}\left(\pi_{1} p_{1}+\sum_{j=1}^{\infty} j\left(1-\pi_{j}\right) p_{j}\right), & j=1 .\end{cases}
$$

Since $\tilde{n}_{j} \leq n_{j}$ for $j \geq 2$ and $\tilde{n} \geq n-n_{0}$, the uniform summability of $j n_{j} / n$ implies uniform summability of $j \tilde{n}_{j} / \tilde{n}$, and thus also

$$
\frac{\sum_{j=0}^{\infty} j \tilde{n}_{j}}{\tilde{n}} \stackrel{\mathrm{p}}{\longrightarrow} \tilde{\lambda}:=\sum_{j=0}^{\infty} \mathrm{j} \tilde{p}_{j}<\infty .
$$

Hence Condition 2.1 holds, in probability, for the random degree sequence $\tilde{\mathbf{d}}$ too. Further, the total number of half-edges is not changed by the explosions, and thus also, by (2.14) and (2.2),

$$
\frac{\sum_{j=0}^{\infty} j \tilde{n}_{j}}{\tilde{n}}=\frac{\sum_{j=0}^{\infty} j n_{j}}{\tilde{n}}=\frac{n}{\tilde{n}} \cdot \frac{\sum_{j=0}^{\infty} j n_{j}}{n} \stackrel{\mathrm{p}}{\longrightarrow} \zeta^{-1} \lambda ;
$$


hence (or by (2.15)),

$$
\tilde{\lambda}=\zeta^{-1} \lambda
$$

In the proofs below it will be convenient to assume that (2.15) and (2.16) hold a.s., and not just in probability, so that Condition 2.1 a.s. holds for $\tilde{\mathbf{d}}$; we can assume this without loss of generality by the Skorohod coupling theorem [13, Theorem 4.30]. (Alternatively, one can argue by selecting suitable subsequences.)

Let $\tilde{D}$ have the probability distribution $\left(\tilde{p}_{j}\right)$, and let $g_{\tilde{D}}$ be its probability generating function. Then, by (2.15),

$$
\zeta g_{\tilde{D}}(x)=\sum_{j=0}^{\infty} \zeta \tilde{p}_{j} x^{j}=\sum_{j=0}^{\infty} \pi_{j} p_{j} x^{j}+\sum_{j=0}^{\infty} j\left(1-\pi_{j}\right) p_{j} x=\lambda x+\sum_{j=0}^{\infty} \pi_{j} p_{j}\left(x^{j}-j x\right) .
$$

In particular, if all $\pi_{j}=\pi$,

$$
\zeta g_{\tilde{D}}(x)=\pi g_{D}(x)+(1-\pi) \lambda x
$$

where now $\zeta=\pi+(1-\pi) \lambda$.

\subsection{Bond percolation}

For bond percolation, we have explosions that do not destroy the vertices, but they may reduce their degrees. Let $\tilde{n}_{l j}$ be the number of vertices that had degree $l$ before the explosions and $j$ after. Thus $\tilde{n}_{j}=\sum_{l \geq j} \tilde{n}_{l j}$ for $j \neq 1$ and $\tilde{n}_{1}=\sum_{l \geq 1} \tilde{n}_{l 1}+n_{+}$. A vertex of degree $l$ will after the explosions have a degree with the binomial distribution $\operatorname{Bi}\left(l, \pi^{1 / 2}\right)$, and thus the probability that it will become a vertex of degree $j$ is the binomial probability $b_{l j}\left(\pi^{1 / 2}\right)$, where we define

$$
b_{l j}(p):=\mathbb{P}(\operatorname{Bi}(l, p)=j)=\left(\begin{array}{l}
l \\
j
\end{array}\right) p^{j}(1-p)^{l-j} .
$$

Since explosions at different vertices occur independently, this means that, for $l \geq j \geq 0$,

$$
\tilde{n}_{l j} \sim \operatorname{Bi}\left(n_{l}, b_{l j}\left(\pi^{1 / 2}\right)\right)
$$

and thus, by the law of large numbers and (2.1),

$$
\tilde{n}_{l j}=b_{l j}\left(\pi^{1 / 2}\right) p_{l} n+o_{p}(n)
$$

Further, the number $n_{+}$of new vertices equals the number of explosions, and thus has the binomial distribution $\operatorname{Bi}\left(\sum_{l} l n_{l}, 1-\pi^{1 / 2}\right)$. Consequently, using also (2.2) and the uniform summability of $\sum_{j} j n_{j} / n$,

$$
\begin{aligned}
& n_{+}=\sum_{l} l_{l}\left(1-\pi^{1 / 2}\right)+o_{p}(n)=\left(1-\pi^{1 / 2}\right) \lambda n+o_{p}(n), \\
& \tilde{n}_{j}=\sum_{l \geq j} \tilde{n}_{l j}=\sum_{l \geq j} b_{l j}\left(\pi^{1 / 2}\right) p_{l} n+o_{p}(n), \quad j \neq 1, \\
& \tilde{n}_{1}=\sum_{l \geq 1} \tilde{n}_{l 1}+n_{+}=\sum_{l \geq 1} b_{l 1}\left(\pi^{1 / 2}\right) p_{l} n+\left(1-\pi^{1 / 2}\right) \lambda n+o_{p}(n),
\end{aligned}
$$




$$
\tilde{n}=n+n_{+}=n+\left(1-\pi^{1 / 2}\right) \lambda n+o_{p}(n) .
$$

In analogy with site percolation we thus have, by (2.25),

$$
\frac{\tilde{n}}{n} \stackrel{\mathrm{p}}{\longrightarrow} \zeta:=1+\left(1-\pi^{1 / 2}\right) \lambda
$$

and further, by (2.23) and (2.24),

$$
\frac{\tilde{n}_{j}}{\tilde{n}} \stackrel{\mathrm{p}}{\longrightarrow} \tilde{p}_{j}:= \begin{cases}\zeta^{-1} \sum_{l \geq j} b_{l j}\left(\pi^{1 / 2}\right) p_{l}, & j \neq 1, \\ \zeta^{-1}\left(\sum_{l \geq 1} b_{l 1}\left(\pi^{1 / 2}\right) p_{l}+\left(1-\pi^{1 / 2}\right) \lambda\right), & j=1 .\end{cases}
$$

Again, the uniform summability of $j n_{j} / n$ implies uniform summability of $j \tilde{n}_{j} / \tilde{n}$, and the total number of half-edges is not changed; thus (2.16), (2.17) and (2.18) hold, now with $\zeta$ given by (2.26). Hence Condition 2.1 holds in probability for the degree sequences $\tilde{\mathbf{d}}$ in bond percolation too, and by the Skorohod coupling theorem we may assume that it holds a.s.

The formula for $\tilde{p}_{j}$ is a bit complicated, but there is a simple formula for the probability generating function $g_{\tilde{D}}$. We have, by the binomial theorem, $\sum_{j \leq l} b_{l j}(\pi) x^{j}=(1-\pi+\pi x)^{l}$, and thus (2.27) yields

$$
\begin{aligned}
\zeta g_{\tilde{D}}(x) & =\sum_{l=0}^{\infty}\left(1-\pi^{1 / 2}+\pi^{1 / 2} x\right)^{l} p_{l}+\left(1-\pi^{1 / 2}\right) \lambda x \\
& =g_{D}\left(1-\pi^{1 / 2}+\pi^{1 / 2} x\right)+\left(1-\pi^{1 / 2}\right) \lambda x .
\end{aligned}
$$

\section{Giant component}

The question of existence of a giant component in $G(n, \mathbf{d})$ and $G^{*}(n, \mathbf{d})$ was answered by Molloy and Reed [16], who showed that (under some weak technical assumptions) a giant component exists w.h.p. if and only if (in the notation above) $\mathbb{E} D(D-2)>0$. (The term giant component is in this paper used, somewhat informally, for a component containing at least a fraction $\varepsilon$ of all vertices, for some small $\varepsilon>0$ that does not depend on $n$.) They further gave a formula for the size of this giant component in Molloy and Reed [15]. We will use the following version of their result, given by Janson and Luczak [12], Theorem 2.3 and Remark 2.6. Let, for any graph $G, \mathscr{C}_{k}(G)$ denote the $k$ :th largest component of $G$. (Break ties by any rule. If there are fewer that $k$ components, let $\mathscr{C}_{k}:=\emptyset$, the null graph.)

Theorem 3.1 ([15; 12]). Consider $G^{*}(n, \mathbf{d})$, assuming that Condition 2.1 holds and $p_{1}>0$. Let $\mathscr{C}_{k}:=\mathscr{C}_{k}\left(G^{*}(n, \mathrm{~d})\right)$ and let $g_{D}(x)$ be the probability generating function in (2.3).

(i) If $\mathbb{E} D(D-2)=\sum_{j} j(j-2) p_{j}>0$, then there is a unique $\xi \in(0,1)$ such that $g_{D}^{\prime}(\xi)=\lambda \xi$, and

$$
\begin{aligned}
& v\left(\mathscr{C}_{1}\right) / n \stackrel{\mathrm{p}}{\longrightarrow} 1-g_{D}(\xi)>0, \\
& v_{j}\left(\mathscr{C}_{1}\right) / n \stackrel{\mathrm{p}}{\longrightarrow} p_{j}\left(1-\xi^{j}\right), \text { for every } j \geq 0, \\
& e\left(\mathscr{C}_{1}\right) / n \stackrel{\mathrm{p}}{\longrightarrow} \frac{1}{2} \lambda\left(1-\xi^{2}\right) .
\end{aligned}
$$

Furthermore, $v\left(\mathscr{C}_{2}\right) / n \stackrel{\mathrm{p}}{\longrightarrow} 0$ and $e\left(\mathscr{C}_{2}\right) / n \stackrel{\mathrm{p}}{\longrightarrow} 0$. 
(ii) If $\mathbb{E} D(D-2)=\sum_{j} j(j-2) p_{j} \leq 0$, then $v\left(\mathscr{C}_{1}\right) / n \stackrel{\mathrm{p}}{\longrightarrow} 0$ and $e\left(\mathscr{C}_{1}\right) / n \stackrel{\mathrm{p}}{\longrightarrow} 0$.

Remark 3.2. $\mathbb{E} D^{2}=\infty$ is allowed in Theorem 3.1(i).

Remark 3.3. In Theorem 3.1(ii), where $\mathbb{E} D(D-2) \leq 0$ and $p_{1}>0$, for $0 \leq x<1$

$$
\begin{aligned}
\lambda x-g_{D}^{\prime}(x) & =\sum_{j=1}^{\infty} j p_{j}\left(x-x^{j-1}\right)=p_{1}(x-1)+x \sum_{j=2}^{\infty} j p_{j}\left(1-x^{j-2}\right) \\
& \leq p_{1}(x-1)+x \sum_{j=2}^{\infty} j p_{j}(j-2)(1-x) \\
& <\sum_{j=1}^{\infty} j(j-2) p_{j} x(1-x)=\mathbb{E} D(D-2) x(1-x) \leq 0 .
\end{aligned}
$$

Hence, in this case the only solution in $[0,1]$ to $g_{D}^{\prime}(\xi)=\lambda \xi$ is $\xi=1$, which we may take as the definition in this case.

Remark 3.4. Let $D^{*}$ be a random variable with the distribution

$$
\mathbb{P}\left(D^{*}=j\right)=(j+1) \mathbb{P}(D=j+1) / \lambda, \quad j \geq 0 ;
$$

this is the size-biased distribution of $D$ shifted by 1 , and it has a well-known natural interpretation as follows. Pick a random half-edge; then the number of remaining half-edges at its endpoint has asymptotically the distribution of $D^{*}$. Therefore, the natural (Galton-Watson) branching process approximation of the exploration of the successive neighbourhoods of a given vertex is the branching process $\overline{\mathscr{X}}$ with offspring distributed as $D^{*}$, but starting with an initial distribution given by $D$. Since

$$
g_{D^{*}}(x)=\sum_{j=1}^{\infty} \mathbb{P}\left(D^{*}=j-1\right) x^{j-1}=\sum_{j=1}^{\infty} \frac{j p_{j}}{\lambda} x^{j-1}=\frac{g_{D}^{\prime}(x)}{\lambda},
$$

the equation $g_{D}^{\prime}(\xi)=\lambda \xi$ in Theorem 3.1(i) can be written $g_{D^{*}}(\xi)=\xi$, which shows that $\xi$ has an interpretation as the extinction probability of the branching process $\mathscr{X}$ with offspring distribution $D^{*}$, now starting with a single individual. (This also agrees with the definition in Remark 3.3 for the case Theorem 3.1(ii).) Thus $g_{D}(\xi)$ in (3.1) is the extinction probability of $\overline{\mathscr{X}}$. Note also that

$$
\mathbb{E} D^{*}=\frac{\mathbb{E} D(D-1)}{\lambda}=\frac{\mathbb{E} D(D-1)}{\mathbb{E} D},
$$

so the condition $\mathbb{E} D(D-2)>0$, or equivalently $\mathbb{E} D(D-1)>\mathbb{E} D$, is equivalent to $\mathbb{E} D^{*}>1$, the classical condition for the branching process to be supercritical and thus have a positive survival probability.

The intuition behind the branching process approximation of the local structure of a random graph at a given vertex is that an infinite approximating branching process corresponds to the vertex being in a giant component. This intuition agrees also with the formulas (3.2) and (3.3), which reflect the fact that a vertex of degree $j$ [an edge] belongs to the giant component if and only if one of its $j$ attached half-edges [one of its two constituent half-edges] connects to the giant component. (It is rather easy to base rigorous proofs on the branching process approximation, see e.g. [4], but in the present paper we will only use the branching process heuristically.) 
Consider one of our percolation models $G^{*}(n, \mathbf{d})_{\pi}$, and construct it using explosions and an intermediate random graph $G^{*}(\tilde{n}, \tilde{\mathbf{d}})$ as described in the introduction. (Recall that $\tilde{\mathbf{d}}$ is random, while $\mathbf{d}$ and the limiting probabilities $p_{j}$ and $\tilde{p}_{j}$ are not.) Let $\mathscr{C}_{j}:=\mathscr{C}_{j}\left(G^{*}(n, \mathbf{d})_{\pi}\right)$ and $\tilde{\mathscr{C}}_{j}:=\mathscr{C}_{j}\left(G^{*}(\tilde{n}, \tilde{\mathbf{d}})\right)$ denote the components of $G^{*}(n, \mathbf{d})_{\pi}$, and $G^{*}(\tilde{n}, \tilde{\mathbf{d}})$, respectively.

As remarked in Section 2, we may assume that $G^{*}(\tilde{n}, \tilde{\mathbf{d}})$ too satisfies Condition 2.1 , with $p_{j}$ replaced by $\tilde{p}_{j}$. (At least a.s.; recall that $\tilde{\mathbf{d}}$ is random.) Hence, assuming $\tilde{p}_{1}>0$, if we first condition on $\tilde{\mathbf{d}}$, then Theorem 3.1 applies immediately to the exploded graph $G^{*}(\tilde{n}, \tilde{\mathbf{d}})$. We also have to remove $n_{+}$randomly chosen "red" vertices of degree 1 , but luckily this will not break up any component. Consequently, if $\mathbb{E} \tilde{D}(\tilde{D}-2)>0$, then $G^{*}(\tilde{n}, \tilde{\mathbf{d}})$ w.h.p. has a giant component $\tilde{\mathscr{C}}_{1}$, with $v\left(\tilde{\mathscr{C}}_{1}\right), v_{j}\left(\tilde{\mathscr{C}}_{1}\right)$ and $e\left(\tilde{\mathscr{C}}_{1}\right)$ given by Theorem 3.1 (with $p_{j}$ replaced by $\tilde{p}_{j}$ ), and after removing the red vertices, the remainder of $\tilde{\mathscr{C}}_{1}$ is still connected and forms a component $\mathscr{C}$ in $G^{*}(n, \mathbf{d})_{\pi}$. Furthermore, since $\mathbb{E} \tilde{D}(\tilde{D}-2)>0, \tilde{p}_{j}>0$ for at least one $j>2$, and it follows by (3.2) that $\tilde{\mathscr{C}}_{1}$ contains $c n+$ $o_{p}(n)$ vertices of degree $j$, for some $c>0$; all these belong to $\mathscr{C}$ (although possibly with smaller degrees), so $\mathscr{C}$ contains w.h.p. at least $c n / 2$ vertices. Moreover, all other components of $G^{*}(n, \mathbf{d})_{\pi}$ are contained in components of $G^{*}(\tilde{n}, \tilde{\mathbf{d}})$ different from $\tilde{\mathscr{C}}_{1}$, and thus at most as large as $\tilde{\mathscr{C}}_{2}$, which by Theorem 3.1 has $o_{p}(\tilde{n})=o_{p}(n)$ vertices. Hence, w.h.p. $\mathscr{C}$ is the largest component $\mathscr{C}_{1}$ of $G^{*}(n, \mathbf{d})_{\pi}$, and this is the unique giant component in $G^{*}(n, \mathbf{d})_{\pi}$.

Since we remove a fraction $n_{+} / \tilde{n}_{1}$ of all vertices of degree 1 , we remove by the law of large numbers (for a hypergeometric distribution) about the same fraction of the vertices of degree 1 in the giant component $\tilde{\mathscr{C}}_{1}$. More precisely, by (3.2), $\tilde{\mathscr{C}}_{1}$ contains about a fraction $1-\xi$ of all vertices of degree 1 , where $g_{\tilde{D}}^{\prime}(\xi)=\tilde{\lambda} \xi$; hence the number of red vertices removed from $\tilde{\mathscr{C}}_{1}$ is

$$
(1-\xi) n_{+}+o_{p}(n)
$$

By (3.1) and (3.4),

$$
v\left(\mathscr{C}_{1}\right)=v\left(\tilde{\mathscr{C}_{1}}\right)-(1-\xi) n_{+}+o_{p}(n)=\tilde{n}\left(1-g_{\tilde{D}}(\xi)\right)-n_{+}+n_{+} \xi+o_{p}(n) .
$$

Similarly, by (3.3) and (3.4), since each red vertex that is removed from $\mathscr{C}_{1}$ also removes one edge with it,

$$
e\left(\mathscr{C}_{1}\right)=e\left(\tilde{\mathscr{C}}_{1}\right)-(1-\xi) n_{+}+o_{p}(n)=\frac{1}{2} \tilde{\lambda} \tilde{n}\left(1-\xi^{2}\right)-(1-\xi) n_{+}+o_{p}(n) .
$$

The case $\mathbb{E} \tilde{D}(\tilde{D}-2) \leq 0$ is even simpler; since the largest component $\mathscr{C}_{1}$ is contained in some component $\tilde{\mathscr{C}}_{j}$ of $G^{*}(\tilde{n}, \tilde{\mathbf{d}})$, it follows that $v\left(\mathscr{C}_{1}\right) \leq v\left(\tilde{\mathscr{C}}_{j}\right) \leq v\left(\tilde{\mathscr{C}}_{1}\right)=o_{p}(\tilde{n})=o_{p}(n)$.

This leads to the following results, where we treat site and bond percolation separately and add formulas for the asymptotic size of $\mathscr{C}_{1}$.

Theorem 3.5. Consider the site percolation model $G^{*}(n, \mathbf{d})_{\pi, v}$, and suppose that Condition 2.1 holds and that $\pi=\left(\pi_{d}\right)_{0}^{\infty}$ with $0 \leq \pi_{d} \leq 1$; suppose further that there exists $j \geq 1$ such that $p_{j}>0$ and $\pi_{j}<1$. Then there is w.h.p. a giant component if and only if

$$
\sum_{j=0}^{\infty} j(j-1) \pi_{j} p_{j}>\lambda:=\sum_{j=0}^{\infty} j p_{j} .
$$

(i) If (3.7) holds, then there is a unique $\xi=\xi_{\mathrm{v}}(\pi) \in(0,1)$ such that

$$
\sum_{j=1}^{\infty} j \pi_{j} p_{j}\left(1-\xi^{j-1}\right)=\lambda(1-\xi)
$$


and then

$$
\begin{aligned}
& v\left(\mathscr{C}_{1}\right) / n \stackrel{\mathrm{p}}{\longrightarrow} \chi_{\mathrm{v}}(\pi):=\sum_{j=1}^{\infty} \pi_{j} p_{j}\left(1-\xi^{j}\right)>0, \\
& e\left(\mathscr{C}_{1}\right) / n \stackrel{\mathrm{p}}{\longrightarrow} \mu_{\mathrm{v}}(\pi):=(1-\xi) \sum_{j=1}^{\infty} j \pi_{j} p_{j}-\frac{(1-\xi)^{2}}{2} \sum_{j=1}^{\infty} j p_{j} .
\end{aligned}
$$

Furthermore, $v\left(\mathscr{C}_{2}\right) / n \stackrel{\mathrm{p}}{\longrightarrow} 0$ and $e\left(\mathscr{C}_{2}\right) / n \stackrel{\mathrm{p}}{\longrightarrow} 0$.

(ii) If (3.7) does not hold, then $v\left(\mathscr{C}_{1}\right) / n \stackrel{\mathrm{p}}{\longrightarrow} 0$ and $e\left(\mathscr{C}_{1}\right) / n \stackrel{\mathrm{p}}{\longrightarrow} 0$.

Proof. We apply Theorem 3.1 to $G^{*}(\tilde{n}, \tilde{\mathbf{d}})$ as discussed above. Note that $\tilde{p}_{1}>0$ by (2.15) and the assumption $\left(1-\pi_{j}\right) p_{j}>0$ for some $j$. By (2.15),

$$
\begin{aligned}
\zeta \mathbb{E} \tilde{D}(\tilde{D}-2) & =\zeta \sum_{j=0}^{\infty} j(j-2) \tilde{p}_{j}=\sum_{j=1}^{\infty} j(j-2) \pi_{j} p_{j}-\sum_{j=1}^{\infty} j\left(1-\pi_{j}\right) p_{j} \\
& =\sum_{j=1}^{\infty} j(j-1) \pi_{j} p_{j}-\sum_{j=1}^{\infty} j p_{j} .
\end{aligned}
$$

Hence, the condition $\mathbb{E} \tilde{D}(\tilde{D}-2)>0$ is equivalent to (3.7).

In particular, it follows that $v\left(\mathscr{C}_{2}\right)=o_{p}(n)$ in (i) and $v\left(\mathscr{C}_{1}\right)=o_{p}(n)$ in (ii). That also $e\left(\mathscr{C}_{2}\right)=o_{p}(n)$ in (i) and $e\left(\mathscr{C}_{1}\right)=o_{p}(n)$ in (ii) follows by Remark 2.2 applied to $G^{*}(\tilde{n}, \tilde{\mathbf{d}})$.

It remains only to verify the formulas (3.8)-(3.10). The equation $g_{\tilde{D}}^{\prime}(\xi)=\tilde{\lambda} \xi$ is by (2.18) equivalent to $\zeta g_{\tilde{D}}^{\prime}(\xi)=\lambda \xi$, which can be written as (3.8) by (2.15) and a simple calculation.

By (3.5), using (2.10), (2.14) and (2.19),

$$
\begin{aligned}
v\left(\mathscr{C}_{1}\right) / n & \stackrel{\mathrm{p}}{\longrightarrow} \zeta-\zeta g_{\tilde{D}}(\xi)-(1-\xi) \sum_{j=1}^{\infty} j\left(1-\pi_{j}\right) p_{j} \\
& =\sum_{j=0}^{\infty} \pi_{j} p_{j}-\sum_{j=0}^{\infty}\left(\pi_{j} p_{j} \xi^{j}+j\left(1-\pi_{j}\right) p_{j} \xi\right)+\xi \sum_{j=0}^{\infty} j\left(1-\pi_{j}\right) p_{j} \\
& =\sum_{j=0}^{\infty} \pi_{j} p_{j}\left(1-\xi^{j}\right) .
\end{aligned}
$$

Similarly, by (3.6), (2.18), (2.14) and (2.10),

$$
\begin{gathered}
e\left(\mathscr{C}_{1}\right) / n \stackrel{\mathrm{p}}{\longrightarrow} \frac{1}{2} \lambda\left(1-\xi^{2}\right)-(1-\xi) \sum_{j=1}^{\infty} j\left(1-\pi_{j}\right) p_{j} \\
=(1-\xi) \sum_{j=1}^{\infty} j \pi_{j} p_{j}-\frac{(1-\xi)^{2}}{2} \lambda
\end{gathered}
$$

In the standard case when all $\pi_{d}=\pi$, this leads to a simple criterion, which earlier has been shown by Britton, Janson and Martin-Löf [4] and Fountoulakis [7] by different methods. (A modification of the usual branching process argument for $G^{*}(n, \mathbf{d})$ in [4] and a method similar to ours in [7].) 
Corollary $3.6([4 ; 7])$. Suppose that Condition 2.1 holds and $0<\pi<1$. Then there exists w.h.p. a giant component in $G^{*}(n, \mathbf{d})_{\pi, v}$ if and only if

$$
\pi>\pi_{\mathrm{c}}:=\frac{\mathbb{E} D}{\mathbb{E} D(D-1)}
$$

Remark 3.7. Note that $\pi_{\mathrm{c}}=0$ is possible; this happens if and only if $\mathbb{E} D^{2}=\infty$. (Recall that we assume $0<\mathbb{E} D<\infty$, see Condition 2.1.) Further, $\pi_{\mathrm{c}} \geq 1$ is possible too: in this case there is w.h.p. no giant component in $G^{*}(n, \mathbf{d})$ (except possibly in the special case when $p_{j}=0$ for all $j \neq 0,2$ ), and consequently none in the subgraph $G^{*}(n, \mathbf{d})_{\pi}$.

Note that by (3.11), $\pi_{\mathrm{c}} \in(0,1)$ if and only if $\mathbb{E} D<\mathbb{E} D(D-1)<\infty$, i.e., if and only if $0<$ $\mathbb{E} D(D-2)<\infty$.

Remark 3.8. Another case treated in [4] (there called E1) is $\pi_{d}=\alpha^{d}$ for some $\alpha \in(0,1)$. Theorem 3.5 gives a new proof that then there is a giant component if and only if $\sum_{j=1}^{\infty} j(j-1) \alpha^{j} p_{j}>\lambda$, which also can be written $\alpha^{2} g_{D}^{\prime \prime}(\alpha)>\lambda=g_{D}^{\prime}(1)$. (The cases E2 and A in [4] are more complicated and do not follow from the results in the present paper.)

For edge percolation we similarly have the following; this too has been shown by Britton, Janson and Martin-Löf [4] and Fountoulakis [7]. Note that the percolation threshold $\pi$ is the same for site and bond percolation, as observed by Fountoulakis [7].

Theorem 3.9 ([4; 7]). Consider the bond percolation model $G^{*}(n, \mathbf{d})_{\pi, \mathrm{e}}$, and suppose that Condition 2.1 holds and that $0<\pi<1$. Then there is w.h.p. a giant component if and only if

$$
\pi>\pi_{\mathrm{c}}:=\frac{\mathbb{E} D}{\mathbb{E} D(D-1)}
$$

(i) If (3.12) holds, then there is a unique $\xi=\xi_{\mathrm{e}}(\pi) \in(0,1)$ such that

$$
\pi^{1 / 2} g_{D}^{\prime}\left(1-\pi^{1 / 2}+\pi^{1 / 2} \xi\right)+\left(1-\pi^{1 / 2}\right) \lambda=\lambda \xi
$$

and then

$$
\begin{aligned}
& v\left(\mathscr{C}_{1}\right) / n \stackrel{\mathrm{p}}{\longrightarrow} \chi_{\mathrm{e}}(\pi):=1-g_{D}\left(1-\pi^{1 / 2}+\pi^{1 / 2} \xi\right)>0, \\
& e\left(\mathscr{C}_{1}\right) / n \stackrel{\mathrm{p}}{\longrightarrow} \mu_{\mathrm{e}}(\pi):=\pi^{1 / 2}(1-\xi) \lambda-\frac{1}{2} \lambda(1-\xi)^{2} .
\end{aligned}
$$

Furthermore, $v\left(\mathscr{C}_{2}\right) / n \stackrel{\mathrm{p}}{\longrightarrow} 0$ and $e\left(\mathscr{C}_{2}\right) / n \stackrel{\mathrm{p}}{\longrightarrow} 0$.

(ii) If (3.12) does not hold, then $v\left(\mathscr{C}_{1}\right) / n \stackrel{\mathrm{p}}{\longrightarrow} 0$ and $e\left(\mathscr{C}_{1}\right) / n \stackrel{\mathrm{p}}{\longrightarrow} 0$.

Proof. We argue as in the proof of Theorem 3.5, noting that $\tilde{p}_{1}>0$ by (2.27). By (2.28),

$$
\begin{aligned}
\zeta \mathbb{E} \tilde{D}(\tilde{D}-2) & =\zeta g_{\tilde{D}}^{\prime \prime}(1)-\zeta g_{\tilde{D}}^{\prime}(1)=\pi g_{D}^{\prime \prime}(1)-\pi^{1 / 2} g_{D}^{\prime}(1)-\left(1-\pi^{1 / 2}\right) \lambda \\
& =\pi \mathbb{E} D(D-1)-\lambda,
\end{aligned}
$$

which yields the criterion (3.12). Further, if (3.12) holds, then the equation $g_{\tilde{D}}^{\prime}(\xi)=\tilde{\lambda} \xi$, which by (2.18) is equivalent to $\zeta g_{\tilde{D}}^{\prime}(\xi)=\zeta \tilde{\lambda} \xi=\lambda \xi$, becomes (3.13) by (2.28). 
By (3.5), (2.26), (2.22) and (2.28),

$$
\left.v\left(\mathscr{C}_{1}\right) / n \stackrel{\mathrm{p}}{\longrightarrow} \zeta-\zeta g_{\tilde{D}}(\xi)-(1-\xi)\left(1-\pi^{1 / 2}\right) \lambda=1-g_{D}\left(1-\pi^{1 / 2}+\pi^{1 / 2} \xi\right)\right),
$$

which is (3.14). Similarly, (3.6), (2.26), (2.18) and (2.22) yield

$$
e\left(\mathscr{C}_{1}\right) / n \stackrel{\mathrm{p}}{\longrightarrow} \frac{1}{2} \lambda\left(1-\xi^{2}\right)-(1-\xi)\left(1-\pi^{1 / 2}\right) \lambda=\pi^{1 / 2}(1-\xi) \lambda-\frac{1}{2} \lambda(1-\xi)^{2},
$$

which is (3.15). The rest is as above.

Remark 3.10. It may come as a surprise that we have the same criterion (3.11) and (3.12) for site and bond percolation, since the proofs above arrive at this equation in somewhat different ways. However, remember that all results here are consistent with the standard branching process approximation in Remark 3.4 (even if our proofs use different arguments) and it is obvious that both site and bond percolation affect the mean number of offspring in the branching process in the same way, namely by multiplication by $\pi$. Cf. [4], where the proofs are based on such branching process approximations.

Define

$$
\rho_{\mathrm{v}}=\rho_{\mathrm{v}}(\pi):=1-\xi_{\mathrm{v}}(\pi) \quad \text { and } \quad \rho_{\mathrm{e}}=\rho_{\mathrm{e}}(\pi):=1-\xi_{\mathrm{e}}(\pi) ;
$$

recall from Remark 3.4 that $\xi_{v}$ and $\xi_{\mathrm{e}}$ are the extinction probabilities in the two branching processes defined by the site and bond percolation models, and thus $\rho_{\mathrm{v}}$ and $\rho_{\mathrm{e}}$ are the corresponding survival probabilities. For bond percolation, (3.13)-(3.15) can be written in the somewhat simpler forms

$$
\begin{aligned}
\pi^{1 / 2} g_{D}^{\prime}\left(1-\pi^{1 / 2} \rho_{\mathrm{e}}\right) & =\lambda\left(\pi^{1 / 2}-\rho_{\mathrm{e}}\right), \\
v\left(\mathscr{C}_{1}\right) / n \stackrel{\mathrm{p}}{\longrightarrow} \chi_{\mathrm{e}}(\pi) & :=1-g_{D}\left(1-\pi^{1 / 2} \rho_{\mathrm{e}}(\pi)\right), \\
e\left(\mathscr{C}_{1}\right) / n \stackrel{\mathrm{p}}{\longrightarrow} \mu_{\mathrm{e}}(\pi) & :=\pi^{1 / 2} \lambda \rho_{\mathrm{e}}(\pi)-\frac{1}{2} \lambda \rho_{\mathrm{e}}(\pi)^{2} .
\end{aligned}
$$

Note further that if we consider site percolation with all $\pi_{j}=\pi$, (3.8) can be written

$$
\pi\left(\lambda-g_{D}^{\prime}\left(1-\rho_{\mathrm{v}}\right)\right)=\lambda \rho_{\mathrm{v}}
$$

and it follows by comparison with (3.17) that

$$
\rho_{\mathrm{v}}(\pi)=\pi^{1 / 2} \rho_{\mathrm{e}}(\pi)
$$

Furthermore, (3.9), (3.10), (3.18) and (3.19) now yield

$$
\begin{aligned}
& \chi_{\mathrm{v}}(\pi)=\pi\left(1-g_{D}\left(\xi_{\mathrm{v}}(\pi)\right)\right)=\pi\left(1-g_{D}\left(1-\rho_{\mathrm{v}}(\pi)\right)\right)=\pi \chi_{\mathrm{e}}(\pi), \\
& \mu_{\mathrm{v}}(\pi)=\pi \lambda \rho_{\mathrm{v}}(\pi)-\frac{1}{2} \lambda \rho_{\mathrm{v}}(\pi)^{2}=\pi \mu_{\mathrm{e}}(\pi) .
\end{aligned}
$$

We next consider how the various parameters above depend on $\pi$, for both site percolation and bond percolation, where for site percolation we in the remainder of this section consider only the case when all $\pi_{j}=\pi$.

We have so far defined the parameters for $\pi \in\left(\pi_{c}, 1\right)$ only; we extend the definitions by letting $\xi_{\mathrm{v}}:=\xi_{\mathrm{e}}:=1$ and $\rho_{\mathrm{v}}:=\rho_{\mathrm{e}}:=\chi_{\mathrm{v}}:=\chi_{\mathrm{e}}:=\mu_{\mathrm{v}}:=\mu_{\mathrm{e}}:=0$ for $\pi \leq \pi_{\mathrm{c}}$, noting that this is compatible with the branching process interpretation of $\xi$ and $\rho$ in Remark 3.4 and that the equalities in (3.8)(3.23) hold trivially. 
Theorem 3.11. Assume Condition 2.1. The functions $\xi_{\mathrm{v}}, \rho_{\mathrm{v}}, \chi_{\mathrm{v}}, \mu_{\mathrm{v}}, \xi_{\mathrm{e}}, \rho_{\mathrm{e}}, \chi_{\mathrm{e}}, \mu_{\mathrm{e}}$ are continuous functions of $\pi \in(0,1)$ and are analytic except at $\pi=\pi_{c}$. (Hence, the functions are analytic in $(0,1)$ if and only if $\pi_{\mathrm{c}}=0$ or $\pi_{\mathrm{c}} \geq 1$.)

Proof. It suffices to show this for $\xi_{v}$; the result for the other functions then follows by (3.16) and (3.21)-(3.23). Since the case $\pi \leq \pi_{c}$ is trivial, it suffices to consider $\pi \geq \pi_{c}$, and we may thus assume that $0 \leq \pi_{\mathrm{c}}<1$.

If $\pi \in\left(\pi_{\mathrm{c}}, 1\right)$, then, as shown above, $g_{\tilde{D}}^{\prime}\left(\xi_{\mathrm{v}}\right)=\tilde{\lambda} \xi_{\mathrm{v}}$, or, equivalently, $G\left(\xi_{\mathrm{v}}, \pi\right)=0$, where $G(\xi, \pi):=$ $g_{\tilde{D}}^{\prime}(\xi) / \xi-\tilde{\lambda}$ is an analytic function of $(\xi, \pi) \in(0,1)^{2}$. Moreover, $G(\xi, \pi)$ is a strictly convex function of $\xi \in(0,1]$ for any $\pi \in(0,1)$, and $G\left(\xi_{\mathrm{v}}, \pi\right)=G(1, \pi)=0$; hence $\left.\frac{\partial G(\xi, \pi)}{\partial \xi}\right|_{\xi=\xi_{\mathrm{v}}}<0$. The implicit function theorem now shows that $\xi_{\mathrm{v}}(\pi)$ is analytic for $\pi \in\left(\pi_{\mathrm{c}}, 1\right)$.

For continuity at $\pi_{\mathrm{c}}$, suppose $\pi_{\mathrm{c}} \in(0,1)$ and let $\hat{\xi}=\lim _{n \rightarrow \infty} \xi_{\mathrm{v}}\left(\pi_{n}\right)$ for some sequence $\pi_{n} \rightarrow \pi_{\mathrm{c}}$. Then, writing $\tilde{D}(\pi)$ and $\tilde{\lambda}(\pi)$ to show the dependence on $\pi, g_{\tilde{D}\left(\pi_{n}\right)}^{\prime}\left(\xi_{\mathrm{v}}\left(\pi_{n}\right)\right)=\tilde{\lambda}\left(\pi_{n}\right) \xi_{\mathrm{v}}\left(\pi_{n}\right)$ and thus by continuity, e.g. using (2.28), $g_{\tilde{D}\left(\pi_{c}\right)}^{\prime}(\hat{\xi})=\tilde{\lambda}\left(\pi_{c}\right) \hat{\xi}$. However, for $\pi \leq \pi_{c}$, we have $\mathbb{E} \tilde{D}(\tilde{D}-2) \leq 0$ and then $\xi=1$ is the only solution in $(0,1]$ of $g_{\tilde{D}}^{\prime}(\xi)=\tilde{\lambda} \xi$; hence $\hat{\xi}=1$. This shows that $\xi_{\mathrm{v}}(\pi) \rightarrow 1$ as $\pi \rightarrow \pi_{c}$, i.e., $\xi_{v}$ is continuous at $\pi_{c}$.

Remark 3.12. Alternatively, the continuity of $\xi_{v}$ in $(0,1)$ follows by Remark 3.4 and continuity of the extinction probability as the offspring distribution varies, cf. [4, Lemma 4.1]. Furthermore, by the same arguments, the parameters are continuous also at $\pi=0$ and, except in the case when $p_{0}+p_{2}=1$ (and thus $\tilde{D}=1$ a.s.), at $\pi=1$ too.

At the threshold $\pi_{c}$, we have linear growth of the size of the giant component for (slightly) larger $\pi$, provided $\mathbb{E} D^{3}<\infty$, and thus a jump discontinuity in the derivative of $\xi_{\mathrm{v}}, \chi_{\mathrm{v}}, \ldots$. More precisely, the following holds. We are here only interested in the case $0<\pi_{\mathrm{c}}<1$, which is equivalent to $0<\mathbb{E} D(D-2)<\infty$, see Remark 3.7.

Theorem 3.13. Suppose that $0<\mathbb{E} D(D-2)<\infty$; thus $0<\pi_{\mathrm{c}}<1$. If further $\mathbb{E} D^{3}<\infty$, then as $\varepsilon \searrow 0$,

$$
\begin{aligned}
& \rho_{\mathrm{v}}\left(\pi_{\mathrm{c}}+\varepsilon\right) \sim \frac{2 \mathbb{E} D(D-1)}{\pi_{\mathrm{c}} \mathbb{E} D(D-1)(D-2)} \varepsilon=\frac{2(\mathbb{E} D(D-1))^{2}}{\mathbb{E} D \cdot \mathbb{E} D(D-1)(D-2)} \varepsilon \\
& \chi_{\mathrm{v}}\left(\pi_{\mathrm{c}}+\varepsilon\right) \sim \mu_{\mathrm{v}}\left(\pi_{\mathrm{c}}+\varepsilon\right) \sim \pi_{\mathrm{c}} \lambda \rho_{\mathrm{v}}\left(\pi_{\mathrm{c}}+\varepsilon\right) \sim \frac{2 \mathbb{E} D \cdot \mathbb{E} D(D-1)}{\mathbb{E} D(D-1)(D-2)} \varepsilon .
\end{aligned}
$$

Similar results for $\rho_{\mathrm{e}}, \chi_{\mathrm{e}}, \mu_{\mathrm{e}}$ follow by (3.21)-(3.23).

Proof. For $\pi=\pi_{\mathrm{c}}+\varepsilon \searrow \pi_{\mathrm{c}}$, by $g_{D}^{\prime \prime}(1)=\mathbb{E} D(D-1)=\lambda / \pi_{\mathrm{c}}$, see (3.11), and (3.20),

$$
\varepsilon g_{D}^{\prime \prime}(1) \rho_{\mathrm{v}}=\left(\pi-\pi_{\mathrm{c}}\right) g_{D}^{\prime \prime}(1) \rho_{\mathrm{v}}=\pi g_{D}^{\prime \prime}(1) \rho_{\mathrm{v}}-\lambda \rho_{\mathrm{v}}=\pi\left(g_{D}^{\prime \prime}(1) \rho_{\mathrm{v}}-\lambda+g_{D}^{\prime}\left(1-\rho_{\mathrm{v}}\right)\right) \text {. }
$$

Since $\mathbb{E} D^{3}<\infty, g_{D}$ is three times continuously differentiable on $[0,1]$, and a Taylor expansion yields $g_{D}^{\prime}\left(1-\rho_{\mathrm{v}}\right)=\lambda-\rho_{\mathrm{v}} g_{D}^{\prime \prime}(1)+\rho_{\mathrm{v}}^{2} g_{D}^{\prime \prime \prime}(1) / 2+o\left(\rho_{\mathrm{v}}^{2}\right)$. Hence, (3.26) yields, since $\rho_{\mathrm{v}}>0$,

$$
\varepsilon g_{D}^{\prime \prime}(1)=\pi \rho_{\mathrm{v}} g_{D}^{\prime \prime \prime}(1) / 2+o\left(\rho_{\mathrm{v}}\right)=\pi_{\mathrm{c}} \rho_{\mathrm{v}} g_{D}^{\prime \prime \prime}(1) / 2+o\left(\rho_{\mathrm{v}}\right) .
$$


Thus, noting that $g_{D}^{\prime \prime}(1)=\mathbb{E} D(D-1)$ and $g_{D}^{\prime \prime \prime}(1)=\mathbb{E} D(D-1)(D-2)>0($ since $\mathbb{E} D(D-2)>0)$,

$$
\rho_{\vee} \sim \frac{2 g_{D}^{\prime \prime}(1)}{\pi_{\mathrm{c}} g_{D}^{\prime \prime \prime}(1)} \varepsilon=\frac{2 \mathbb{E} D(D-1)}{\pi_{\mathrm{c}} \mathbb{E} D(D-1)(D-2)} \varepsilon,
$$

which yields (3.24). Finally, (3.25) follows easily by (3.22) and (3.23).

If $\mathbb{E} D^{3}=\infty$, we find in the same way a slower growth of $\rho_{\mathrm{v}}(\pi), \chi_{\mathrm{v}}(\pi), \mu_{\mathrm{v}}(\pi)$ at $\pi_{\mathrm{c}}$. As an example, we consider $D$ with a power law tail, $p_{k} \sim c k^{-\gamma}$, where we take $3<\gamma<4$ so that $\mathbb{E} D^{2}<\infty$ but $\mathbb{E} D^{3}=\infty$.

Theorem 3.14. Suppose that $p_{k} \sim c k^{-\gamma}$ as $k \rightarrow \infty$, where $3<\gamma<4$ and $c>0$. Assume further that $\mathbb{E} D(D-2)>0$. Then $\pi_{\mathrm{c}} \in(0,1)$ and, as $\varepsilon \searrow 0$,

$$
\begin{aligned}
\rho_{\mathrm{v}}\left(\pi_{\mathrm{c}}+\varepsilon\right) & \sim\left(\frac{\mathbb{E} D(D-1)}{c \pi_{\mathrm{c}} \Gamma(2-\gamma)}\right)^{1 /(\gamma-3)} \varepsilon^{1 /(\gamma-3)}, \\
\chi_{\mathrm{v}}\left(\pi_{\mathrm{c}}+\varepsilon\right) & \sim \mu_{\mathrm{v}}\left(\pi_{\mathrm{c}}+\varepsilon\right) \sim \pi_{\mathrm{c}} \lambda \rho_{\mathrm{v}}\left(\pi_{\mathrm{c}}+\varepsilon\right) \\
& \sim \pi_{\mathrm{c}} \lambda\left(\frac{\mathbb{E} D(D-1)}{c \pi_{\mathrm{c}} \Gamma(2-\gamma)}\right)^{1 /(\gamma-3)} \varepsilon^{1 /(\gamma-3)} .
\end{aligned}
$$

Similar results for $\rho_{\mathrm{e}}, \chi_{\mathrm{e}}, \mu_{\mathrm{e}}$ follow by (3.21)-(3.23).

Proof. We have, for example by comparison with the Taylor expansion of $(1-(1-t))^{\gamma-4}$,

$$
g_{D}^{\prime \prime \prime}(1-t)=\sum_{k=3}^{\infty} k(k-1)(k-2) p_{k}(1-t)^{k-3} \sim c \Gamma(4-\gamma) t^{\gamma-4}, \quad t \searrow 0
$$

and thus by integration

$$
g_{D}^{\prime \prime}(1)-g_{D}^{\prime \prime}(1-t) \sim c \Gamma(4-\gamma)(\gamma-3)^{-1} t^{\gamma-3}=c|\Gamma(3-\gamma)| t^{\gamma-3},
$$

and, integrating once more,

$$
\rho_{\vee} g_{D}^{\prime \prime}(1)-\left(\lambda-g_{D}^{\prime}\left(1-\rho_{\vee}\right)\right) \sim c \Gamma(2-\gamma) \rho_{\vee}^{\gamma-2}
$$

Hence, (3.26) yields

$$
\varepsilon g_{D}^{\prime \prime}(1) \rho_{\mathrm{v}} \sim c \pi_{\mathrm{c}} \Gamma(2-\gamma) \rho_{\mathrm{v}}^{\gamma-2}
$$

and the results follow, again using (3.22) and (3.23).

\section{$4 k$-core}

Let $k \geq 2$ be a fixed integer. The $k$-core of a graph $G$, denoted by $\operatorname{Core}_{k}(G)$, is the largest induced subgraph of $G$ with minimum vertex degree at least $k$. (Note that the $k$-core may be empty.) The question whether a non-empty $k$-core exists in a random graph has attracted a lot of attention for various models of random graphs since the pioneering papers by Bollobás [2], Łuczak [14] and Pittel, Spencer and Wormald [17] for $G(n, p)$ and $G(n, m)$; in particular, the case of $G(n, \mathbf{d})$ and 
$G^{*}(n, \mathbf{d})$ with given degree sequences have been studied by several authors, see Janson and Luczak $[10,11]$ and the references given there.

We study the percolated $G^{*}(n, \mathbf{d})_{\pi}$ by the exposion method presented in Section 1 . For the $k$-core, the cleaning up stage is trivial: by definition, the $k$-core of $G^{*}(\tilde{n}, \tilde{\mathbf{d}})$ does not contain any vertices of degree 1 , so it is unaffected by the removal of all red vertices, and thus

$$
\operatorname{Core}_{k}\left(G^{*}(n, \mathbf{d})_{\pi}\right)=\operatorname{Core}_{k}\left(G^{*}(\tilde{n}, \tilde{\mathbf{d}})\right) .
$$

Let, for $0 \leq p \leq 1, D_{p}$ be the thinning of $D$ obtained by taking $D$ points and then randomly and independently keeping each of them with probability $p$. Thus, given $D=d, D_{p} \sim \operatorname{Bi}(d, p)$. Define, recalling the notation (2.21),

$$
\begin{aligned}
h(p) & :=\mathbb{E}\left(D_{p} \mathbf{1}\left[D_{p} \geq k\right]\right)=\sum_{j=k}^{\infty} \sum_{l=j}^{\infty} j p_{l} b_{l j}(p), \\
h_{1}(p) & :=\mathbb{P}\left(D_{p} \geq k\right)=\sum_{j=k}^{\infty} \sum_{l=j}^{\infty} p_{l} b_{l j}(p) .
\end{aligned}
$$

Note that $D_{p}$ is stochastically increasing in $p$, and thus both $h$ and $h_{1}$ are increasing in $p$, with $h(0)=h_{1}(0)=0$. Note further that $h(1)=\sum_{j=k}^{\infty} j p_{j} \leq \lambda$ and $h_{1}(1)=\sum_{j=k}^{\infty} p_{j} \leq 1$, with strict inequalities unless $p_{j}=0$ for all $j=1, \ldots, k-1$ or $j=0,1, \ldots, k-1$, respectively. Moreover,

$$
\begin{aligned}
h(p) & =\mathbb{E} D_{p}-\mathbb{E}\left(D_{p} \mathbf{1}\left[D_{p} \leq k-1\right]\right)=\mathbb{E} D_{p}-\sum_{j=1}^{k-1} j \mathbb{P}\left(D_{p}=j\right) \\
& =\lambda p-\sum_{j=1}^{k-1} \sum_{l \geq j} j p_{l}\left(\begin{array}{l}
l \\
j
\end{array}\right) p^{j}(1-p)^{l-j} \\
& =\lambda p-\sum_{j=1}^{k-1} \frac{p^{j}}{(j-1) !} g_{D}^{(j)}(1-p) .
\end{aligned}
$$

Since $g_{D}(z)$ is an analytic function in $\{z:|z|<1\}$, (4.4) shows that $h(p)$ is an analytic function in the domain $\{p:|p-1|<1\}$ in the complex plane; in particular, $h$ is analytic on $(0,1]$. (But not necessarily at 0, as seen by Example 4.13.) Similarly, $h_{1}$ is analytic on $(0,1]$.

We will use the following result by Janson and Luczak [10], Theorem 2.3.

Theorem 4.1 ([10]). Suppose that Condition 2.1 holds. Let $k \geq 2$ be fixed, and let Core ${ }_{k}^{*}$ be the $k$-core of $G^{*}(n, \mathbf{d})$. Let $\widehat{p}:=\max \left\{p \in[0,1]: h(p)=\lambda p^{2}\right\}$.

(i) If $h(p)<\lambda p^{2}$ for all $p \in(0,1]$, which is equivalent to $\widehat{p}=0$, then $\operatorname{Core}_{k}^{*}$ has $o_{p}(n)$ vertices and $o_{p}(n)$ edges. Furthermore, if also $k \geq 3$ and $\sum_{i=1}^{n} e^{\alpha d_{i}}=O(n)$ for some $\alpha>0$, then Core ${ }_{k}^{*}$ is empty w.h.p.

(ii) If $h(p) \geq \lambda p^{2}$ for some $p \in(0,1]$, which is equivalent to $\widehat{p} \in(0,1]$, and further $\widehat{p}$ is not a local maximum point of $h(p)-\lambda p^{2}$, then

$$
v\left(\operatorname{Core}_{k}^{*}\right) / n \stackrel{\mathrm{p}}{\longrightarrow} h_{1}(\widehat{p})>0,
$$




$$
\begin{aligned}
& v_{j}\left(\text { Core }_{k}^{*}\right) / n \stackrel{\mathrm{p}}{\longrightarrow} \mathbb{P}\left(D_{\widehat{p}}=j\right)=\sum_{l=j}^{\infty} p_{l} b_{l j}(\widehat{p}), \quad j \geq k, \\
& e\left(\text { Core }_{k}^{*}\right) / n \stackrel{\mathrm{p}}{\longrightarrow} h(\widehat{p}) / 2=\lambda \widehat{p}^{2} / 2 .
\end{aligned}
$$

Remark 4.2. The result (4.6) is not stated explicitly in [10], but as remarked in [11, Remark 1.8], it follows immediately from the proof in [10] of (4.5). (Cf. [5] for the random graph $G(n, m)$.)

Remark 4.3. The extra condition in (ii) that $\widehat{p}$ is not a local maximum point of $h(p)-\lambda p^{2}$ is actually stated somewhat differently in [10], viz. as $\lambda p^{2}<h(p)$ in some interval $(\widehat{p}-\varepsilon, \widehat{p})$. However, since $g(p):=h(p)-\lambda p^{2}$ is analytic at $\widehat{p}$, a Taylor expansion at $\widehat{p}$ shows that either $g(p)=0$ for all $p$ (and then $\widehat{p}=1)$, or for some such interval $(\widehat{p}-\varepsilon, \widehat{p})$, either $g(p)>0$ or $g(p)<0$ throughout the interval. Since $g(\widehat{p})=0$ and $g(p)<0$ for $\widehat{p}<p \leq 1$, the two versions of the condition are equivalent.

The need for this condition is perhaps more clearly seen in the percolation setting, cf. Remark 4.8.

There is a natural interpretation of this result in terms of the branching process approximation of the local exploration process, similar to the one described for the giant component in Remark 3.4. For the $k$-core, this was observed already by Pittel, Spencer and Wormald [17], but (unlike for the giant component), the branching process approximation has so far mainly been used heuristically; the technical difficulties to make a rigorous proof based on it are formidable, and have so far been overcome only by Riordan [18] for a related random graph model. We, as most others, avoid this complicated method of proof, and only identify the limits in Theorem 4.1 (which is proved by other, simpler, methods in [10]) with quantities for the branching process. Although this idea is not new, we have, for the random graphs that we consider, not seen a detailed proof of it in the literature, so for completeness we provide one in Appendix A.

Remark 4.4. If $k=2$, then (4.4) yields

$$
h(p)=\lambda p-\sum_{l=0}^{\infty} p_{l} l p(1-p)^{l-1}=\lambda p-p g_{D}^{\prime}(1-p)
$$

and thus

$$
h(p)-\lambda p^{2}=p\left(\lambda(1-p)-g_{D}^{\prime}(1-p)\right) .
$$

It follows that $\widehat{p}=1-\xi$, where $\xi$ is as in Theorem 3.1 and Remark 3.3 ; i.e., by Remark $3.4, \widehat{p}=\rho$, the survival probability of the branching process $\mathscr{X}$ with offspring distribution $D^{*}$. (See Appendix $\mathrm{A}$ for further explanations of this.)

We now easily derive results for the $k$-core in the percolation models. For simplicity, we consider for site percolation only the case when all $\pi_{k}$ are equal; the general case is similar but the explicit formulas are less nice.

Theorem 4.5. Consider the site percolation model $G^{*}(n, \mathbf{d})_{\pi, v}$ with $0 \leq \pi \leq 1$, and suppose that Condition 2.1 holds. Let $k \geq 2$ be fixed, and let Core $_{k}^{*}$ be the $k$-core of $G^{*}(n, \mathbf{d})_{\pi, \mathrm{v}}$. Let

$$
\pi_{c}=\pi_{c}^{(k)}:=\inf _{0<p \leq 1} \frac{\lambda p^{2}}{h(p)}=\left(\sup _{0<p \leq 1} \frac{h(p)}{\lambda p^{2}}\right)^{-1} .
$$


(i) If $\pi<\pi_{c}$, then Core ${ }_{k}^{*}$ has $o_{p}(n)$ vertices and $o_{p}(n)$ edges. Furthermore, if also $k \geq 3$ and $\sum_{i=1}^{n} e^{\alpha d_{i}}=O(n)$ for some $\alpha>0$, then Core ${ }_{k}^{*}$ is empty w.h.p.

(ii) If $\pi>\pi_{c}$, then w.h.p. Core ${ }_{k}^{*}$ is non-empty. Furthermore, if $\widehat{p}=\widehat{p}(\pi)$ is the largest $p \leq 1$ such that $h(p) /\left(\lambda p^{2}\right)=\pi^{-1}$, and $\widehat{p}$ is not a local maximum point of $h(p) /\left(\lambda p^{2}\right)$ in $(0,1]$, then

$$
\begin{aligned}
& v\left(\operatorname{Core}_{k}^{*}\right) / n \stackrel{\mathrm{p}}{\longrightarrow} \pi h_{1}(\widehat{p})>0, \\
& v_{j}\left(\operatorname{Core}_{k}^{*}\right) / n \stackrel{\mathrm{p}}{\longrightarrow} \pi \mathbb{P}\left(D_{\widehat{p}}=j\right), \quad j \geq k, \\
& e\left(\operatorname{Core}_{k}^{*}\right) / n \stackrel{\mathrm{p}}{\longrightarrow} \pi h(\widehat{p}) / 2=\lambda \widehat{p}^{2} / 2 .
\end{aligned}
$$

Theorem 4.6. Consider the bond percolation model $G^{*}(n, \mathbf{d})_{\pi, \mathrm{e}}$ with $0 \leq \pi \leq 1$, and suppose that Condition 2.1 holds. Let $k \geq 2$ be fixed, and let Core ${ }_{k}^{*}$ be the $k$-core of $G^{*}(n, \mathbf{d})_{\pi, \mathrm{e}}$. Let $\pi_{\mathrm{c}}=\pi_{\mathrm{c}}^{(k)}$ be given by (4.9).

(i) If $\pi<\pi_{c}$, then Core ${ }_{k}^{*}$ has $o_{p}(n)$ vertices and $o_{p}(n)$ edges. Furthermore, if also $k \geq 3$ and $\sum_{i=1}^{n} e^{\alpha d_{i}}=O(n)$ for some $\alpha>0$, then Core ${ }_{k}^{*}$ is empty w.h.p.

(ii) If $\pi>\pi_{c}$, then w.h.p. Core $_{k}^{*}$ is non-empty. Furthermore, if $\widehat{p}=\widehat{p}(\pi)$ is the largest $p \leq 1$ such that $h(p) /\left(\lambda p^{2}\right)=\pi^{-1}$, and $\widehat{p}$ is not a local maximum point of $h(p) /\left(\lambda p^{2}\right)$ in $(0,1]$, then

$$
\begin{aligned}
& v\left(\operatorname{Core}_{k}^{*}\right) / n \stackrel{\mathrm{p}}{\longrightarrow} h_{1}(\widehat{p})>0, \\
& v_{j}\left(\operatorname{Core}_{k}^{*}\right) / n \stackrel{\mathrm{p}}{\longrightarrow} \mathbb{P}\left(D_{\widehat{p}}=j\right), \quad j \geq k, \\
& e\left(\operatorname{Core}_{k}^{*}\right) / n \stackrel{\mathrm{p}}{\longrightarrow} h(\widehat{p}) / 2=\lambda \widehat{p}^{2} /(2 \pi) .
\end{aligned}
$$

For convenience, we define $\varphi(p):=h(p) / p^{2}, 0<p \leq 1$.

Remark 4.7. Since $h(p)$ is analytic in $(0,1)$, there is at most a countable number of local maximum points of $\varphi(p):=h(p) / p^{2}$ (except when $h(p) / p^{2}$ is constant), and thus at most a countable number of local maximum values of $h(p) / p^{2}$. Hence, there is at most a countable number of exceptional values of $\pi$ in part (ii) of Theorems 4.5 and 4.6. At these exceptional values, we have a discontinuity of $\widehat{p}(\pi)$ and thus of the relative asymptotic size $\pi h_{1}(\widehat{p}(\pi))$ or $h_{1}(\widehat{p}(\pi))$ of the $k$-core; in other words, there is a phase transition of the $k$-core at each such exceptional $\pi$. (See Figures 1 and 2.) Similarly, if $\varphi$ has an inflection point at $\widehat{p}(\pi)$, i.e., if $\varphi^{\prime}(\widehat{p})=\varphi^{\prime \prime}(\widehat{p})=\cdots=\varphi^{(2 \ell)}(\widehat{p})=0$ and $\varphi^{(2 \ell+1)}(\widehat{p})<0$ for some $\ell \geq 1$, then $\widehat{p}(\pi)$ and $h_{1}(\widehat{p}(\pi))$ are continuous but the derivatives of $\widehat{p}(\pi)$ and $h_{1}(\widehat{p}(\pi))$ become infinite at this point, so we have a phase transition of a different type. For all other $\pi>\pi_{\mathrm{c}}$, the implicit function theorem shows that $\widehat{p}(\pi)$ and $h_{1}(\widehat{p}(\pi))$ are analytic at $\pi$.

Say that $\tilde{p}$ is a critical point of $\varphi$ if $\varphi^{\prime}(\tilde{p})=0$, and a bad critical point if further, $\tilde{p} \in(0,1), \varphi(\tilde{p})>\lambda$ and $\varphi(\tilde{p})>\varphi(p)$ for all $p \in(\tilde{p}, 1)$. It follows that there is a 1-1 correspondence between phase transitions in $\left(\pi_{c}, 1\right)$ or $\left[\pi_{c}, 1\right)$ and bad critical points $\tilde{p}$ of $\varphi$, with the phase transition occurring at $\tilde{\pi}=\lambda / \varphi(\tilde{p})$. This includes $\pi_{c}$ if and only if $\sup _{(0,1]} \varphi(p)$ is attained and larger than $\lambda$, in which case the last global maximum point is a bad critical point; if this supremum is finite not attained, then there is another first-order phase transition at $\pi_{c}$, while if the supremum is infinite, then $\pi_{c}=0$. Finally, there may be a further phase transition at $\tilde{\pi}=1$ (with $\tilde{p}=1$ ); this happens if and only if $\varphi(1)=h(1)=\lambda$ and $\varphi^{\prime}(1) \leq 0$. 
The phase transitions are first-order when the corresponding $\tilde{p}$ is a bad local maximum point of $\varphi$, i.e., a bad critical point that is a local maximum point. (This includes $\pi_{c}$ when $\sup _{(0,1]} \varphi$ is attained, but not otherwise.) Thus, the phase transition that occur are typically first order, but there are exceptions, see Examples 4.13 and 4.18 .

Remark 4.8. The behaviour at $\pi=\pi_{\mathrm{c}}$ depends on more detailed properties of the degree sequences $\left(d_{i}^{(n)}\right)_{1}^{n}$, or equivalently of $\hat{D}_{n}$. Indeed, more precise results can be derived from Janson and Luczak [11], Theorem 3.5, at least under somewhat stricter conditions on $\left(d_{i}^{(n)}\right)_{1}^{n}$; in particular, it then follows that the width of the threshold is of the order $n^{-1 / 2}$, i.e., that there is a sequence $\pi_{c n}$ depending on $\left(d_{i}^{(n)}\right)_{1}^{n}$, with $\pi_{c n} \rightarrow \pi_{c}$, such that $G^{*}(n, \mathbf{d})_{\pi, v}$ and $G^{*}(n, \mathbf{d})_{\pi, \mathrm{e}}$ w.h.p. have a non-empty $k$-core if $\pi=\pi_{c n}+\omega(n) n^{-1 / 2}$ with $\omega(n) \rightarrow \infty$, but w.h.p. an empty $k$-core if $\pi=\pi_{c n}-\omega(n) n^{-1 / 2}$, while in the intermediate case $\pi=\pi_{c n}+c n^{-1 / 2}$ with $-\infty<c<\infty, \mathbb{P}\left(G^{*}(n, \mathbf{d})_{\pi}\right.$ has a non-empty $k$-core $)$ converges to a limit (depending on $c$ ) in $(0,1)$. We leave the details to the reader.

The same applies to further phase transitions that may occur.

Remark 4.9. If $k=2$, then (4.8) yields

$$
\varphi(p):=h(p) / p^{2}=\sum_{j \geq 2} p_{j} j\left(1-(1-p)^{j-1}\right) / p,
$$

which is decreasing on $(0,1]$ (or constant, when $\mathbb{P}(D>2)=0)$, with

$$
\sup _{p \in(0,1]} \varphi(p)=\lim _{p \rightarrow 0} \varphi(p)=\sum_{j} p_{j} j(j-1)=\mathbb{E} D(D-1) \leq \infty .
$$

Hence

$$
\pi_{\mathrm{c}}^{(2)}=\lambda / \mathbb{E} D(D-1)=\mathbb{E} D / \mathbb{E} D(D-1),
$$

coinciding with the critical value in (3.11) for a giant component.

Although there is no strict implication in any direction between "a giant component" and "a nonempty 2-core", in random graphs these seem to typically appear together (in the form of a large connected component of the 2-core), see Appendix A for branching process heuristics explaing this.

Remark 4.10. We see again that the results for site and bond percolation are almost identical. In fact, they become the same if we measure the size of the $k$-core in relation to the size of the percolated graph $G^{*}(n, \mathbf{d})_{\pi}$, since $v\left(G^{*}(n, \mathbf{d})_{\pi, \mathrm{e}}\right)=n$ but $v\left(G^{*}(n, \mathbf{d})_{\pi, \mathrm{v}}\right) \sim \operatorname{Bi}(n, \pi)$, so $v\left(G^{*}(n, \mathbf{d})_{\pi, v}\right) / n \stackrel{\mathrm{p}}{\longrightarrow} \pi$. Again, this is heuristically explained by the branching process approximations; see Appendix $\mathrm{A}$ and note that random deletions of vertices or edges yield the same result in the branching process, assuming that we do not delete the root.

Proof of Theorem 4.5. The case $\mathbb{P}(D \geq k)=0$ is trivial; in this case $h(p)=0$ for all $p$ and $\pi_{\mathrm{c}}=0$ so (i) applies. Further, Theorem 4.1(i) applies to $G^{*}(n, \mathbf{d})$, and the result follows from Core $_{k}\left(G^{*}(n, \mathbf{d})_{\pi, v}\right) \subseteq \operatorname{Core}_{k}\left(G^{*}(n, \mathbf{d})\right)$. In the sequel we thus assume $\mathbb{P}(D \geq k)>0$, which implies $h(p)>0$ and $h_{1}(p)>0$ for $0<p \leq 1$.

We apply Theorem 4.1 to the exploded graph $G^{*}(\tilde{n}, \tilde{\mathbf{d}})$, recalling (4.1). For site percolation, $G^{*}(n, \mathbf{d})_{\pi, \mathrm{v}}, \tilde{p}_{j}=\zeta^{-1} \pi p_{j}$ for $j \geq 2$ by (2.15), and thus

$$
\mathbb{P}\left(\tilde{D}_{p}=j\right)=\zeta^{-1} \pi \mathbb{P}\left(D_{p}=j\right), \quad j \geq 2,
$$


and, because $k \geq 2$,

$$
\begin{aligned}
\tilde{h}(p) & :=\mathbb{E}\left(\tilde{D}_{p} \mathbf{1}\left[\tilde{D}_{p} \geq k\right]\right)=\zeta^{-1} \pi h(p), \\
\tilde{h}_{1}(p) & :=\mathbb{P}\left(\tilde{D}_{p} \geq k\right)=\zeta^{-1} \pi h_{1}(p) .
\end{aligned}
$$

Hence, the condition $\tilde{h}(p) \geq \tilde{\lambda} p^{2}$ can, using (2.18), be written

$$
\pi h(p) \geq \lambda p^{2} .
$$

If $\pi<\pi_{c}$, then for every $p \in(0,1]$, by (4.9), $\pi<\pi_{c} \leq \lambda p^{2} / h(p)$ so (4.12) does not hold and $\tilde{h}(p)<$ $\tilde{\lambda} p^{2}$. Hence Theorem 4.1(i) applies to $G^{*}(\tilde{n}, \tilde{\mathbf{d}})$, which proves (i); note that if $\sum_{i=1}^{n} e^{\alpha d_{i}}=O(n)$ for some $\alpha>0$, then also

$$
\sum_{i=1}^{\tilde{n}} e^{\alpha \tilde{d}_{i}} \leq \sum_{i=1}^{n} e^{\alpha d_{i}}+n_{+} e^{\alpha} \leq \sum_{i=1}^{n} e^{\alpha d_{i}}+e^{\alpha} \sum_{j \geq 1} j n_{j}=O(n) .
$$

If $\pi>\pi_{c}$, then there exists $p \in(0,1]$ such that $\pi>\lambda p^{2} / h(p)$ and thus (4.12) holds and Theorem 4.1(ii) applies to $G^{*}(\tilde{n}, \tilde{\mathbf{d}})$. Moreover, $\widehat{p}$ in Theorem 4.1(ii) is the largest $p \leq 1$ such that (4.12) holds. Since $\pi h(1) \leq h(1) \leq \lambda$ and $h$ is continuous, we have equality in (4.12) for $p=\widehat{p}$, i.e., $\pi h(\widehat{p})=\lambda \widehat{p}^{2}$, so $\widehat{p}$ is as asserted the largest $p \leq 1$ with $h(p) /\left(\lambda p^{2}\right)=\pi^{-1}$.

Further, if $\widehat{p}$ is a local maximum point of $\tilde{h}(p)-\tilde{\lambda} p^{2}=\zeta^{-1}\left(\pi h(p)-\lambda p^{2}\right)$, then $\pi h(p)-\lambda p^{2} \leq 0$ in a neighbourhood of $\widehat{p}$ and thus $h(p) /\left(\lambda p^{2}\right) \leq 1 / \pi=h(\widehat{p}) /\left(\lambda \widehat{p}^{2}\right)$ there; thus $\widehat{p}$ is a local maximum point of $h(p) /\left(\lambda p^{2}\right)$. Excluding such points, we obtain from Theorem 4.1(ii) using (4.1), (2.14), (2.15), (2.18), (4.10) and (4.11),

$$
\begin{aligned}
\frac{v\left(\operatorname{Core}_{k}^{*}\right)}{n} & =\frac{\tilde{n}}{n} \cdot \frac{v\left(\operatorname{Core}_{k}^{*}\right)}{\tilde{n}} \stackrel{\mathrm{p}}{\longrightarrow} \zeta \tilde{h}_{1}(\widehat{p})=\pi h_{1}(\widehat{p}), \\
\frac{v_{j}\left(\operatorname{Core}_{k}^{*}\right)}{n} & =\frac{\tilde{n}}{n} \cdot \frac{v_{j}\left(\operatorname{Core}_{k}^{*}\right)}{\tilde{n}} \stackrel{\mathrm{p}}{\longrightarrow} \zeta \mathbb{P}\left(\tilde{D}_{\widehat{p}}=j\right)=\pi \mathbb{P}\left(D_{\widehat{p}}=j\right), \quad j \geq k, \\
\frac{e\left(\operatorname{Core}_{k}^{*}\right)}{n} & =\frac{\tilde{n}}{n} \cdot \frac{e\left(\operatorname{Core}_{k}^{*}\right)}{\tilde{n}} \stackrel{\mathrm{p}}{\longrightarrow} \zeta \frac{\tilde{\lambda} \widehat{p}^{2}}{2}=\frac{\lambda \widehat{p}^{2}}{2} .
\end{aligned}
$$

This proves the result when $\widehat{p}$ is not a local maximum point of $h(p) /\left(\lambda p^{2}\right)$. In particular, since $h_{1}(\widehat{p})>0$, Core $_{k}^{*}$ is non-empty w.h.p. when $\pi>\pi_{\mathrm{c}}$ is not a local maximum value of $h(p) /\left(\lambda p^{2}\right)$. Finally, even if $\pi$ is such a local maximum value, we can find $\pi^{\prime}$ with $\pi_{c}<\pi^{\prime}<\pi$ that is not, because by Remark 4.7 there is only a countable number of exceptional $\pi$. By what we just have shown, $G^{*}(n, \mathbf{d})_{\pi^{\prime}, \mathrm{v}}$ has w.h.p. a non-empty $k$-core, and thus so has $G^{*}(n, \mathbf{d})_{\pi, v} \supseteq G^{*}(n, \mathbf{d})_{\pi^{\prime}, \mathrm{v}}$.

Proof of Theorem 4.6. We argue as in the proof just given of Theorem 4.6, again using (4.1) and applying Theorem 4.1 to the exploded graph $G^{*}(\tilde{n}, \tilde{\mathbf{d}})$. We may again assume $\mathbb{P}(D \geq k)>0$, and thus $h(p)>0$ and $h_{1}(p)>0$ for $0<p \leq 1$. We may further assume $\pi>0$.

The main difference from the site percolation case is that for bond percolation $G^{*}(n, \mathbf{d})_{\pi, \mathrm{e}},(2.27)$ yields

$$
\mathbb{P}(\tilde{D}=j)=\zeta^{-1} \mathbb{P}\left(D_{\pi^{1 / 2}}=j\right), \quad j \geq 2,
$$

and hence

$$
\mathbb{P}\left(\tilde{D}_{p}=j\right)=\zeta^{-1} \mathbb{P}\left(D_{p \pi^{1 / 2}}=j\right), \quad j \geq 2,
$$


and thus

$$
\begin{aligned}
\tilde{h}(p) & :=\mathbb{E}\left(\tilde{D}_{p} \mathbf{1}\left[\tilde{D}_{p} \geq k\right]\right)=\zeta^{-1} h\left(p \pi^{1 / 2}\right), \\
\tilde{h}_{1}(p) & :=\mathbb{P}\left(\tilde{D}_{p} \geq k\right)=\zeta^{-1} h_{1}\left(p \pi^{1 / 2}\right) .
\end{aligned}
$$

Consequently, the condition $\tilde{h}(p) \geq \tilde{\lambda} p^{2}$ can, using (2.18), be written as $h\left(p \pi^{1 / 2}\right) \geq \lambda p^{2}$, or

$$
\pi h\left(p \pi^{1 / 2}\right) \geq \lambda\left(p \pi^{1 / 2}\right)^{2} .
$$

If $\pi<\pi_{c}$, then for every $p \in(0,1]$ we have $p \pi^{1 / 2} \in(0,1]$ and thus by (4.9)

$$
\pi<\pi_{\mathrm{c}} \leq \frac{\lambda\left(p \pi^{1 / 2}\right)^{2}}{h\left(p \pi^{1 / 2}\right)}
$$

so (4.16) does not hold and $\tilde{h}(p)<\tilde{\lambda} p^{2}$. Hence Theorem 4.1(i) applies to $G^{*}(\tilde{n}, \tilde{\mathbf{d}})$ as in the proof of Theorem 4.5 .

If $\pi>\pi_{c}$, then there exists $p \in(0,1]$ such that $\pi>\lambda p^{2} / h(p)$ and, as before, $\widehat{p}$ is the largest such $p$ and satisfies $h(\widehat{p}) /\left(\lambda \widehat{p}^{2}\right)=\pi^{-1}$. Furthermore, if $\pi^{1 / 2}<p \leq 1$, then

$$
\pi h(p) \leq \pi h(1) \leq \pi \lambda<\lambda p^{2},
$$

and thus $p \neq \widehat{p}$. Hence $\widehat{p} \leq \pi^{1 / 2}$. Let $\widehat{p}_{0}:=\widehat{p} / \pi^{1 / 2}$. Then $\widehat{p}_{0} \in(0,1]$ and $\widehat{p}_{0}$ is the largest $p \leq 1$ such that (4.16) holds; i.e., the largest $p \leq 1$ such that $\tilde{h}(p) \geq \tilde{\lambda} p^{2}$. We thus can apply Theorem 4.1(ii) to $G^{*}(\tilde{n}, \tilde{\mathbf{d}})$, with $\widehat{p}$ replaced by $\widehat{p}_{0}$, noting that if $\widehat{p}_{0}$ is a local maximum point of $\tilde{h}(p)-\tilde{\lambda} p^{2}$, then $\widehat{p}$ is a local maximum point of

$$
\tilde{h}\left(p \pi^{-1 / 2}\right)-\tilde{\lambda}\left(p \pi^{-1 / 2}\right)^{2}=\zeta^{-1}\left(h(p)-\pi^{-1} \lambda p^{2}\right)
$$

and thus of $\pi h(p)-\lambda p^{2}$, which as in the proof of Theorem 4.5 implies that $\widehat{p}$ is a local maximum point of $h(p) /\left(\lambda p^{2}\right)$. (The careful reader may note that there is no problem with the special case $\widehat{p}_{0}=1$, when we only consider a one-sided maximum at $\widehat{p}_{0}$ : in this case $\widehat{p}=\pi^{1 / 2}$ and $\pi h(\widehat{p})=$ $\lambda \widehat{p}^{2}=\lambda \pi$ so $h(\widehat{p})=\lambda$ and $\widehat{p}=1, \pi=1$.) Consequently, when $\widehat{p}$ is not a local maximum point of $h(p) /\left(\lambda p^{2}\right)$, Theorem 4.1(ii) yields, using (2.26), (4.13), (4.15),

$$
\begin{aligned}
\frac{v\left(\operatorname{Core}_{k}^{*}\right)}{n} & =\frac{\tilde{n}}{n} \cdot \frac{v\left(\operatorname{Core}_{k}^{*}\right)}{\tilde{n}} \stackrel{\mathrm{p}}{\longrightarrow} \zeta \tilde{h}_{1}\left(\widehat{p}_{0}\right)=h_{1}(\widehat{p}), \\
\frac{v_{j}\left(\operatorname{Core}_{k}^{*}\right)}{n} & =\frac{\tilde{n}}{n} \cdot \frac{v_{j}\left(\operatorname{Core}_{k}^{*}\right)}{\tilde{n}} \stackrel{\mathrm{p}}{\longrightarrow} \zeta \mathbb{P}\left(\tilde{D}_{\widehat{p}_{0}}=j\right)=\mathbb{P}\left(D_{\widehat{p}}=j\right), \quad j \geq k, \\
\frac{e\left(\operatorname{Core}_{k}^{*}\right)}{n} & =\frac{\tilde{n}}{n} \cdot \frac{e\left(\operatorname{Core}_{k}^{*}\right)}{\tilde{n}} \stackrel{\mathrm{p}}{\longrightarrow} \zeta \frac{\tilde{\lambda} \widehat{p}_{0}^{2}}{2}=\frac{\lambda \widehat{p}^{2}}{2 \pi} .
\end{aligned}
$$

The proof is completed as before.

Consider now what Theorems 4.5 and 4.6 imply for the $k$-core as $\pi$ increases from 0 to 1 . (We will be somewhat informal; the statements below should be interpreted as asymptotic as $n \rightarrow \infty$ for fixed $\pi$, but we for simplicity omit "w.h.p." and “ $\stackrel{\mathrm{p}}{\longrightarrow}$ ".)

If $k=2$, we have by Remark 4.9 a similar behaviour as for the giant component in Section 3; in the interesting case $0<\pi_{c}<1$, the 2-core is small, $o(n)$, for $\pi<\pi_{c}$ and large, $\Theta(n)$, for $\pi>\pi_{c}$, with 
a relative size $h_{1}(\widehat{p}(\pi))$ that is a continuous function of $\pi$ also at $\pi_{c}$ and analytic everywhere else in $(0,1)$, cf. Theorem 3.11 .

Assume now $k \geq 3$. For the random graph $G(n, p)$ with $p=c / n$, the classical result by Pittel, Spencer and Wormald [17] shows that there is a first-order (=discontinuous) phase transition at some value $c_{k}$; for $c<c_{k}$ the $k$-core is empty and for $c>c_{k}$ it is non-empty and with a relative size $\psi_{k}(c)$ that jumps to a positive value at $c=c_{k}$, and thereafter is analytic. We can see this as a percolation result, choosing a large $\lambda$ and regarding $G(n, c / n)$ as obtained by bond percolation on $G(n, \lambda / n)$ with $\pi=c / \lambda$ for $c \in[0, \lambda] ; G(n, \lambda / n)$ is not exactly a random graph of the type $G^{*}(n, \mathbf{d})$ studied in the present paper, but as said in the introduction, it can be treated by our methods by conditioning on the degree sequence, and it has the asymptotic degree distribution $D \sim \operatorname{Po}(\lambda)$. In this case, see Example 4.11 and Figure 1, $\varphi$ is unimodal, with $\varphi(0)=0$, a maximum at some interior point $p_{0} \in(0,1)$, and $\varphi^{\prime}<0$ on $\left(p_{0}, 1\right)$. This is a typical case; $\varphi$ has these properties for many other degree distributions too (and $k \geq 3$ ), and these properties of $\varphi$ imply by Theorems 4.5 and 4.6 that there is, provided $\varphi\left(p_{0}\right)>\lambda$, a first-order phase transition at $\pi=\pi_{c}=\lambda / \varphi\left(p_{0}\right)$ where the $k$-core suddenly is created with a positive fraction $h_{1}\left(p_{0}\right)$ of all vertices, but no other phase transitions since $h_{1}(\widehat{p}(\pi))$ is analytic on $\left(\pi_{c}, 1\right)$. Equivalently, recalling Remark 4.7, we see that $p_{0}$ is the only bad critical point of $\varphi$.

However, there are other possibilities too; there may be several bad critical points of $\varphi$, and thus several phase transitions of $\varphi$. There may even be an infinite number of them. We give some examples showing different possibilities that may occur. (A similar example with several phase transitions for a related hypergraph process is given by Darling, Levin and Norris [6].)

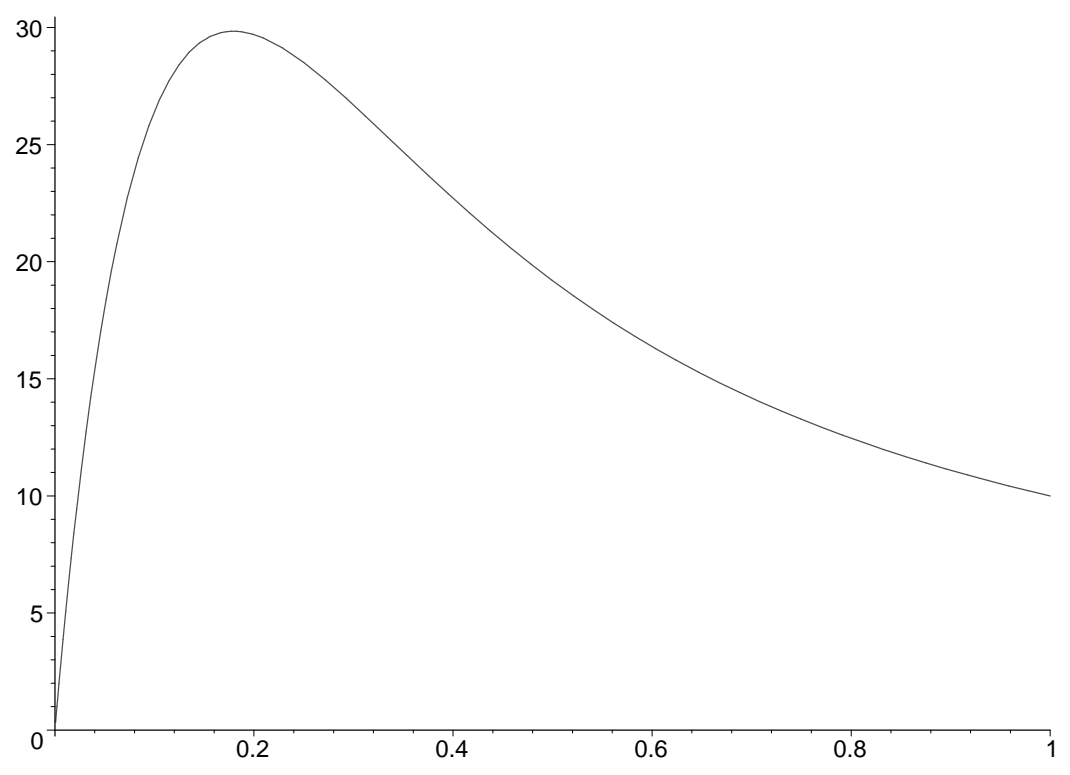

Figure 1: $\varphi(p)=h(p) / p^{2}$ for $D \sim \operatorname{Po}(10)$ and $k=3$.

Example 4.11. A standard case is when $D \sim \operatorname{Po}(\lambda)$ and $k \geq 3$. (This includes, as said above, the case $G(n, \lambda / n)$ by conditioning on the degree sequence, in which case we recover the result by [17].) Then $D_{p} \sim \operatorname{Po}(\lambda p)$ and a simple calculation shows that $h(p)=\lambda p \mathbb{P}(\operatorname{Po}(\lambda p) \geq k-1)$, see $[10$, p. 


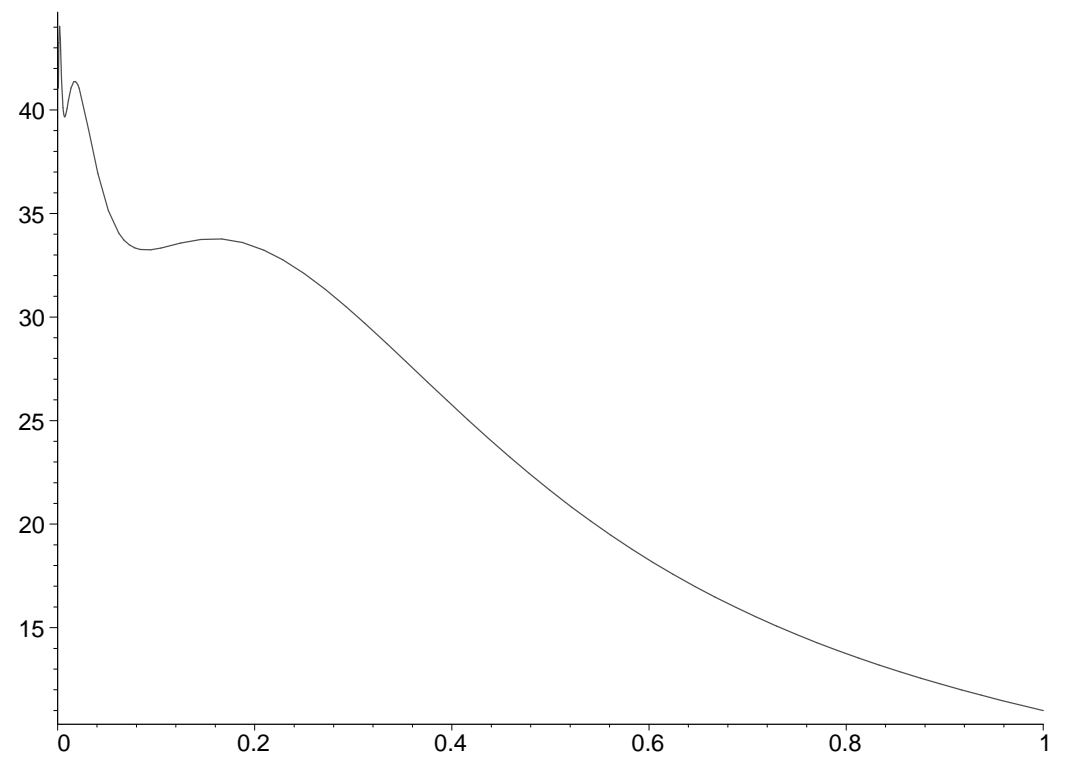

Figure 2: $\varphi(p)=h(p) / p^{2}$ for $k=3$ and $p_{10^{i}}=99 \cdot 10^{-2 i}, i=1,2, \ldots,\left(p_{j}=0\right.$ for all other $\left.j\right)$. Cf. Example 4.15.

59]. Hence, if $c_{k}:=\min _{\mu>0} \mu / \mathbb{P}(\operatorname{Po}(\mu) \geq k-1)$ and $\lambda>c_{k}$, then $\pi_{c}=\inf _{0<p \leq 1}\left(\lambda p^{2} / h(p)\right)=$ $c_{k} / \lambda$. Moreover, it is easily shown that $h(p) / p^{2}$ is unimodal, see [10, Lemma 7.2] and Figure 1. Consequently, there is as discussed above a single first-order phase transition at $\pi=c_{k} / \lambda$ [17].

Example 4.12. Let $k=3$ and consider graphs with only two vertex degrees, 3 and $m$, say, with $m \geq 4$. Then, cf. (4.4),

$$
\begin{aligned}
h(p) & =3 p_{3} \mathbb{P}\left(D_{p}=3 \mid D=3\right)+p_{m} \mathbb{E}\left(D_{p}-D_{p} \mathbf{1}\left[D_{p} \leq 2\right] \mid D=m\right) \\
& =3 p_{3} p^{3}+p_{m}\left(m p-m p(1-p)^{m-1}-m(m-1) p^{2}(1-p)^{m-2}\right) .
\end{aligned}
$$

Now, let $p_{3}:=1-a / m$ and $p_{m}:=a / m$, with $a>0$ fixed and $m \geq a$, and let $m \rightarrow \infty$. Then, writing $h=h_{m}, h_{m}(p) \rightarrow 3 p^{3}+a p$ for $p \in(0,1]$ and thus

$$
\varphi_{m}(p):=\frac{h_{m}(p)}{p^{2}} \rightarrow \varphi_{\infty}(p):=3 p+\frac{a}{p}
$$

Since $\varphi_{\infty}^{\prime}(1)=3-a$, we see that if we choose $a=1$, say, then $\varphi_{\infty}^{\prime}(1)>0$. Furthermore, then $\varphi_{\infty}(1 / 4)=\frac{3}{4}+4>\varphi_{\infty}(1)=4$. Since also $\varphi_{m}^{\prime}(1)=3 p_{3}-m p_{m}=3 p_{3}-a \rightarrow \varphi_{\infty}^{\prime}(1)$, it follows that if $m$ is large enough, then $\varphi_{m}^{\prime}(1)>0$ but $\varphi_{m}(1 / 4)>\varphi_{m}(1)$. We fix such an $m$ and note that $\varphi=\varphi_{m}$ is continuous on $[0,1]$ with $\varphi(0)=0$, because the sum in (4.2) is finite with each term $O\left(p^{3}\right)$.

Let $\tilde{p}_{0}$ be the global maximum point of $\varphi$ in $[0,1]$. (If not unique, take the largest value.) Then, by the properties just shown, $\tilde{p}_{0} \neq 0$ and $\tilde{p}_{0} \neq 1$, so $\tilde{p}_{0} \in(0,1)$; moreover, 1 is a local maximum point but not a global maximum point. Hence, $\pi_{c}=\lambda / \varphi\left(\tilde{p}_{0}\right)$ is a first-order phase transition where the 3-core suddenly becomes non-empty and containing a positive fraction $h_{1}\left(\tilde{p}_{0}\right)$ of all (remaining) vertices. There is another phase transition at $\pi=1$. We have $\varphi(1)=h(1)=\lambda$, but since $\varphi^{\prime}(1)>0$, 
if $\tilde{p}_{1}:=\sup \{p<1: \varphi(p)>\varphi(1)\}$, then $\tilde{p}_{1}<1$. Hence, as $\pi \nearrow 1, \widehat{p}(\pi) \nearrow \tilde{p}_{1}$ and $h(\widehat{p}(\pi)) \nearrow$ $h_{1}\left(\tilde{p}_{1}\right)<1$. Consequently, the size of the 3 -core jumps again at $\pi=1$.

For an explicit example, numerical calculations (using Maple) show that we can take $a=1$ and $m=12$, or $a=1.9$ and $m=6$.

Example 4.13. Let $k=3$ and let $D$ be a mixture of Poisson distributions:

$$
\mathbb{P}(D=j)=p_{j}=\sum_{i} q_{i} \mathbb{P}\left(\operatorname{Po}\left(\lambda_{i}\right)=j\right), \quad j \geq 0,
$$

for some finite or infinite sequences $\left(q_{i}\right)$ and $\left(\lambda_{i}\right)$ with $q_{i} \geq 0, \sum_{i} q_{i}=1$ and $\lambda_{i} \geq 0$. In the case $D \sim \operatorname{Po}(\lambda)$ we have, cf. Example 4.11, $D_{p} \sim \operatorname{Po}(\lambda p)$ and thus

$$
\begin{aligned}
h(p) & =\mathbb{E} D_{p}-\mathbb{P}\left(D_{p}=1\right)-2 \mathbb{P}\left(D_{p}=2\right)=\lambda p-\lambda p e^{-\lambda p}-(\lambda p)^{2} e^{-\lambda p} \\
& =(\lambda p)^{2} f(\lambda p),
\end{aligned}
$$

where $f(x):=\left(1-(1+x) e^{-x}\right) / x$. Consequently, by linearity, for $D$ given by (4.18),

$$
h(p)=\sum_{i} q_{i}\left(\lambda_{i} p\right)^{2} f\left(\lambda_{i} p\right)
$$

and thus

$$
\varphi(p)=\sum_{i} q_{i} \lambda_{i}^{2} f\left(\lambda_{i} p\right)
$$

As a specific example, take $\lambda_{i}=2^{i}$ and $q_{i}=\lambda_{i}^{-2}=2^{-2 i}, i \geq 1$, and add $q_{0}=1-\sum_{i \geq 1} q_{i}$ and $\lambda_{0}=0$ to make $\sum q_{i}=1$. Then

$$
\varphi(p)=\sum_{i=1}^{\infty} f\left(2^{i} p\right) .
$$

Note that $f(x)=O(x)$ and $f(x)=O\left(x^{-1}\right)$ for $0<x<\infty$. Hence, the sum in (4.21) converges uniformly on every compact interval $[\delta, 1]$; moreover, if we define

$$
\psi(x):=\sum_{i=-\infty}^{\infty} f\left(2^{i} x\right),
$$

then the sum converges uniformly on compact intervals of $(0, \infty)$ and $|\varphi(p)-\psi(p)|=O(p)$. Clearly, $\psi$ is a multiplicatively periodic function on $(0, \infty): \psi(2 x)=\psi(x)$; we compute the Fourier series of the periodic function $\psi\left(2^{y}\right)$ on $\mathbb{R}$ and find $\psi\left(2^{y}\right)=\sum_{n=-\infty}^{\infty} \widehat{\psi}(n) e^{2 \pi \text { iny }}$ with, using integration by 
parts,

$$
\begin{aligned}
\widehat{\psi}(n) & =\int_{0}^{1} \psi\left(2^{y}\right) e^{-2 \pi \mathrm{in} y} \mathrm{~d} y=\int_{0}^{1} \sum_{j=-\infty}^{\infty} f\left(2^{j} 2^{y}\right) e^{-2 \pi \mathrm{in} y} \mathrm{~d} y \\
& =\sum_{j=-\infty}^{\infty} \int_{0}^{1} f\left(2^{j+y}\right) e^{-2 \pi \mathrm{in} y} \mathrm{~d} y=\int_{-\infty}^{\infty} f\left(2^{y}\right) e^{-2 \pi \mathrm{in} y} \mathrm{~d} y \\
& =\int_{0}^{\infty} f(x) x^{-2 \pi \mathrm{in} / \ln 2} \frac{\mathrm{d} x}{x \ln 2} \\
& =\frac{1}{\ln 2} \int_{0}^{\infty}\left(1-(1+x) e^{-x}\right) x^{-2 \pi \mathrm{in} / \ln 2-2} \mathrm{~d} x \\
& =\frac{1}{\ln 2(2 \pi \mathrm{i} n / \ln 2+1)} \int_{0}^{\infty} x e^{-x} x^{-2 \pi \mathrm{in} / \ln 2-1} \mathrm{~d} x \\
& =\frac{\Gamma(1-2 \pi \mathrm{i} / \ln 2)}{\ln 2+2 \pi \mathrm{i} n} .
\end{aligned}
$$

Since these Fourier coefficients are non-zero, we see that $\psi$ is a non-constant continuous function on $(0, \infty)$ with multiplicative period 2 . Let $a>0$ be any point that is a global maximum of $\psi$, let $b \in(a, 2 a)$ be a point that is not, and let $I_{j}:=\left[2^{-j-1} b, 2^{-j} b\right]$. Then $\psi$ attains its global maximum at the interior point $2^{-j} a$ in $I_{j}$, and since $\varphi(p)-\psi(p)=O\left(2^{-j}\right)$ for $p \in I_{j}$, it follows that if $j$ is large enough, then $\varphi\left(2^{-j} a\right)>\max \left(\varphi\left(2^{-j-1} b\right), \varphi\left(2^{-j} b\right)\right)$. Hence, if the maximum of $\varphi$ on $I_{j}$ is attained at $\tilde{p}_{j} \in I_{j}$ (choosing the largest maximum point if it is not unique), then, at least for large $j, \tilde{p}_{j}$ is in the interior of $I_{j}$, so $\tilde{p}_{j}$ is a local maximum point of $\varphi$. Further, as $j \rightarrow \infty$, $\varphi\left(\tilde{p}_{j}\right) \rightarrow \max \psi>\widehat{\psi}(0)=1 / \ln 2$ while $\lambda:=\mathbb{E} D=\sum_{i} q_{i} \lambda_{i}=\sum_{1}^{\infty} 2^{-i}=1$, so $\varphi\left(\tilde{p}_{j}\right)>\lambda$ for large $j$. Moreover, $\tilde{p}_{j} / 2 \in I_{j+1}$, and since (4.21) implies

$$
\varphi(p / 2)=\sum_{i=1}^{\infty} f\left(2^{i-1} p\right)=\sum_{i=0}^{\infty} f\left(2^{i} p\right)>\varphi(p), \quad p>0,
$$

thus $\varphi\left(\tilde{p}_{j}\right)<\varphi\left(\tilde{p}_{j} / 2\right) \leq \varphi\left(\tilde{p}_{j+1}\right)$. It follows that if $p \in I_{i}$ for some $i<j$, then $\varphi(p) \leq \varphi\left(\tilde{p}_{i}\right)<\varphi\left(\tilde{p}_{j}\right)$. Consequently, for large $j$ at least, $\tilde{p}_{j}$ is a bad local maximum point, and thus there is a phase transition at $\pi_{j}:=\lambda / \varphi\left(\tilde{p}_{j}\right) \in(0,1)$. This shows that there is an infinite sequence of (first-order) phase transitions.

Further, in this example $\varphi$ is bounded (with $\sup \varphi=\max \psi$ ), and thus $\pi_{c}>0$. Since, by (4.23), $\sup \varphi$ is not attained, this is an example where the phase transition at $\pi_{c}$ is continuous and not firstorder; simple calculations show that as $\pi \searrow \pi_{c}, \widehat{p}(\pi)=\Theta\left(\pi-\pi_{c}\right)$ and $h_{1}(\widehat{p}(\pi))=\Theta\left(\left(\pi-\pi_{c}\right)^{2}\right)$.

Because of the exponential decrease of $|\Gamma(z)|$ on the imaginary axis, $|\widehat{\psi}(n)|$ is very small for $n \neq 0$; we have $|\widehat{\psi}( \pm 1)| \approx 0.78 \cdot 10^{-6}$ and the others much smaller, so $\psi(x)$ deviates from its mean $\widehat{\psi}(0)=$ $1 / \ln 2 \approx 1.44$ by less than $1.6 \cdot 10^{-6}$. The oscillations of $\psi$ and $\varphi$ are thus very small and hard to observe numerically or graphically unless a large precision is used. (Taking e.g. $\lambda_{i}=10^{i}$ yields larger oscillations.)

Note also that in this example, $\varphi$ is not continuous at $p=0 ; \varphi(p)$ is bounded but does not converge as $p \searrow 0$. Thus $h$ is not analytic at $p=0$. 
Example 4.14. Taking $\lambda_{i}=2^{i}$ as in Example 4.13 but modifying $q_{i}$ to $2^{(\varepsilon-2) i}$ for some small $\varepsilon>0$, similar calculations show that $p^{\varepsilon} \varphi(p)=\psi_{\varepsilon}(p)+O(p)$ for a non-constant function $\psi_{\varepsilon}$ with multiplicative period 2, and it follows again that, at least if $\varepsilon$ is small enough, there is an infinite number of phase transitions. In this case, $\varphi(p) \rightarrow \infty$ as $p \rightarrow 0$, so $\pi_{\mathrm{c}}=0$.

Since $\varphi$ is analytic on $(0,1]$, if there is an infinite number of bad critical points, then we may order them (and 1 , if $\varphi^{\prime}(1) \leq 0$ and $\varphi(1)=\lambda$ ) in a decreasing sequence $\tilde{p}_{1}>\tilde{p}_{2}>\ldots$, with $\tilde{p}_{j} \rightarrow 0$. It follows from the definition of bad critical points that then $\varphi\left(\tilde{p}_{1}\right)<\varphi\left(\tilde{p}_{2}\right)<\ldots$, and $\sup _{0<p \leq 1} \varphi(p)=\sup _{j} \varphi\left(\tilde{p}_{j}\right)=\lim _{j \rightarrow \infty} \varphi\left(\tilde{p}_{j}\right)$. Consequently, if there is an infinite number of phase transitions, they occur at $\left\{\pi_{j}\right\}_{1}^{\infty} \cup\left\{\pi_{c}\right\}$ for some decreasing sequence $\pi_{j} \searrow \pi_{c} \geq 0$.

Example 4.15. We can modify 4.13 and 4.14 and consider random graphs where all vertex degrees $d_{i}$ are powers of 2 ; thus $D$ has support on $\left\{2^{i}\right\}$. If we choose $p_{2^{i}} \sim 2^{-2 i}$ or $p_{2^{i}} \sim 2^{(\varepsilon-2) i}$ suitably, the existence of infinitely many phase transitions follows by calculations similar to the ones above. (But the details are a little more complicated, so we omit them.) A similar example concentrated on $\left\{10^{i}\right\}$ is shown in Figure 2 .

Example 4.16. We may modify Example 4.13 by conditioning $D$ on $D \leq M$ for some large $M$. If we denote the corresponding $h$ and $\varphi$ by $h_{M}$ and $\varphi_{M}$, it is easily seen that $h_{M} \rightarrow h$ unformly on $[0,1]$ as $M \rightarrow \infty$, and thus $\varphi_{M} \rightarrow \varphi$ uniformly on every interval $[\delta, 1]$. It follows that if we consider $N$ bad local maximum points of $\varphi$, then there are $N$ corresponding bad local maximum points of $\varphi_{M}$ for large $M$, and thus at least $N$ phase transitions. This shows that we can have any finite number of phase transitions with degree sequences $\left(d_{i}^{(n)}\right)_{1}^{n}$ where the degrees are uniformly bounded. (Example 4.17 shows that we cannot have infinitely many phase transitions in this case.)

Example 4.17. In Examples 4.13 and 4.14 with infinitely many phase transitions, we have $\mathbb{E} D^{2}=$ $\sum_{i} q_{i}\left(\lambda_{i}^{2}+\lambda_{i}\right)=\infty$. This is not a coincidence; in fact, we can show that: If $\mathbb{E} D^{2}<\infty$, then the $k$-core has only finite number of phase transitions.

This is trivial for $k=2$, when there never is more than one phase transition. Thus, assume $k \geq 3$. If $k=3$, then, cf. (4.4),

$$
\begin{aligned}
\varphi(p) & =h(p) / p^{2}=\sum_{l \geq 3} l p_{l}\left(p-p(1-p)^{l-1}-(l-1) p^{2}(1-p)^{l-2}\right) p^{-2} \\
& =\sum_{l \geq 3} l p_{l}\left(\frac{1-(1-p)^{l-1}}{p}-(l-1)(1-p)^{l-2}\right) .
\end{aligned}
$$

Each term in the sum is non-negative and bounded by $l p_{l}\left(1-(1-p)^{l-1}\right) / p \leq l p_{l}(l-1)$, and as $p \rightarrow 0$ it converges to $l p_{l}(l-1-(l-1))=0$. Hence, by dominated convergence, using the assumption $\sum p_{l} l(l-1)=\mathbb{E} D(D-1)<\infty$, we have $\varphi(p) \rightarrow 0$ as $p \rightarrow 0$. For $k>3, h(p)$ is smaller than for $k=3$ (or possibly the same), so we have the same conclusion. Consequently, $\varphi$ is continuous on $[0,1]$ and has a global maximum point $p_{0}$ in $(0,1]$. Every bad critical point has to belong to $\left[p_{0}, 1\right]$. Since $\varphi$ is analytic on $\left[p_{0}, 1\right]$, it has only a finite number of critical points there (except in the trivial case $\varphi(p)=0$ ), and thus there is only a finite number of phase transitions.

Example 4.18. We give an example of a continuous (not first-order) phase transition, letting $D$ be a mixture as in Example 4.13 with two components and carefully chosen weights $q_{1}$ and $q_{2}$.

Let $f$ be as in Example 4.13 and note that $f^{\prime}(x) \sim 1 / 2$ as $x \rightarrow 0$ and $f^{\prime}(x) \sim-x^{-2}$ as $x \rightarrow \infty$. Hence, for some $a, A \in(0, \infty), \frac{1}{4}<f^{\prime}(x)<1$ for $0<x \leq 4 a$ and $\frac{1}{2} x^{-2} \leq-f^{\prime}(x) \leq 2 x^{-2}$ for 
$x \geq A$. Let $f_{1}(x):=f(A x)$ and $f_{2}(x):=f(a x)$. Then, $f_{1}^{\prime}(x)<0$ for $x \geq 1$. Further, if $g(x):=$ $f_{2}^{\prime}(x) /\left|f_{1}^{\prime}(x)\right|=(a / A) f^{\prime}(a x) /\left|f^{\prime}(A x)\right|$, then

$$
\begin{aligned}
& g(1)=\frac{a f^{\prime}(a)}{A\left|f^{\prime}(A)\right|}<\frac{a}{A \cdot A^{-2} / 2}=2 a A, \\
& g(4)=\frac{a f^{\prime}(4 a)}{A\left|f^{\prime}(4 A)\right|}>\frac{a / 4}{A \cdot 2(4 A)^{-2}}=2 a A,
\end{aligned}
$$

and thus $g(1)<g(4)$. Further, if $x \geq A / a$, then $f_{2}^{\prime}(x)<0$ and thus $g(x)<0$. Consequently, $\sup _{x \geq 1} g(x)=\max _{1 \leq x \leq A / a} g(x)<\infty$, and if $x_{0}$ is the point where the latter maximum is attained (choosing the largest value if the maximum is attained at several points), then $1<x_{0}<\infty$ and $g(x)<g\left(x_{0}\right)$ for $x>x_{0}$. Let $\beta:=g\left(x_{0}\right)$ and

$$
\psi(x):=\beta f_{1}(x)+f_{2}(x)=\beta f(A x)+f(a x) .
$$

Then $\psi^{\prime}(x) \leq 0$ for $x \geq 1, \psi^{\prime}\left(x_{0}\right)=0$ and $\psi^{\prime}\left(x_{0}\right)<0$ for $x>x_{0}$.

Let $b$ be large, to be chosen later, and let $D$ be as in Example 4.13 with $q_{1}:=\beta a^{2} /\left(\beta a^{2}+A^{2}\right)$, $q_{2}:=1-q_{1}, \lambda_{1}:=b A, \lambda_{2}:=b a$. Then, by (4.20),

$$
\varphi(p)=q_{1}(b A)^{2} f(b A p)+q_{2}(b a)^{2} f(b a p)=\frac{b^{2} a^{2} A^{2}}{\beta a^{2}+A^{2}} \psi(b p) .
$$

Hence, $\varphi^{\prime}\left(x_{0} / b\right)=0, \varphi^{\prime}(x) \leq 0$ for $x \geq 1 / b$ and $\varphi^{\prime}(x)<0$ for $x>x_{0} / b$. Consequently, $x_{0} / b$ is a critical point but not a local maximum point. Furthermore, $\varphi\left(x_{0} / b\right)=q_{1} b^{2} A^{2} f\left(A x_{0}\right)+$ $q_{2} b^{2} a^{2} f\left(a x_{0}\right)$ and $\lambda:=\mathbb{E} D=q_{1} \lambda_{1}+q_{2} \lambda_{2}=b\left(q_{1} A+q_{2} a\right)$; hence, if $b$ is large enough, then $\varphi\left(x_{0} / b\right)>\lambda$. We choose $b$ such that this holds and $b>x_{0}$; then $\tilde{p}:=x_{0} / b$ is a bad critical point which is an inflection point and not a local maximum point. Hence there is a continuous phase transition at $\tilde{\pi}:=\lambda / \varphi(\tilde{p}) \in\left(\pi_{c}, 1\right)$.

We have $\varphi^{\prime}(\tilde{p})=\varphi^{\prime \prime}(\tilde{p})=0$; we claim that, at least if $A$ is chosen large enough, then $\varphi^{\prime \prime \prime}(\tilde{p}) \neq 0$. This implies that, for some $c_{1}, c_{2}, c_{3}>0, \varphi(p)-\varphi(\tilde{p}) \sim-c_{1}(p-\tilde{p})^{3}$ as $p \rightarrow \tilde{p}$, and $\widehat{p}(\pi)-\widehat{p}(\tilde{\pi}) \sim$ $c_{2}(\pi-\tilde{\pi})^{1 / 3}$ and $h_{1}(\widehat{p}(\pi))-h_{1}(\widehat{p}(\tilde{\pi})) \sim c_{3}(\pi-\tilde{\pi})^{1 / 3}$ as $\pi \rightarrow \tilde{\pi}$, so the critical exponent at $\tilde{\pi}$ is $1 / 3$. To verify the claim, note that if also $\varphi^{\prime \prime \prime}(\tilde{p})=0$, then by (4.25) and (4.24), $\psi^{\prime}\left(x_{0}\right)=\psi^{\prime \prime}\left(x_{0}\right)=$ $\psi^{\prime \prime \prime}\left(x_{0}\right)=0$, and thus

$$
\beta A^{j} f^{(j)}\left(A x_{0}\right)+a^{j} f^{(j)}\left(a x_{0}\right)=0, \quad j=1,2,3 .
$$

Let $x_{1}:=A x_{0}$ and $x_{2}:=a x_{0}$. Then $x_{1} \geq A$, and $f^{\prime}\left(x_{2}\right)>0$ so $x_{2} \leq C$ for some $C$. Further, (4.26) yields

$$
\frac{x_{2} f^{\prime \prime}\left(x_{2}\right)}{f^{\prime}\left(x_{2}\right)}=\frac{x_{1} f^{\prime \prime}\left(x_{1}\right)}{f^{\prime}\left(x_{1}\right)} \quad \text { and } \quad \frac{x_{2}^{2} f^{\prime \prime \prime}\left(x_{2}\right)}{f^{\prime}\left(x_{2}\right)}=\frac{x_{1}^{2} f^{\prime \prime \prime}\left(x_{1}\right)}{f^{\prime}\left(x_{1}\right)} .
$$

Recall that $x_{1}$ and $x_{2}$ depend on our choices of $a$ and $A$, and that we always can decrease $a$ and increase $A$. Keep $a$ fixed and let $A \rightarrow \infty$ (along some sequence). Then $x_{1} \rightarrow \infty$ but $x_{2}=O(1)$, so by selecting a subsequence we may assume $x_{2} \rightarrow y \geq 0$. As $x \rightarrow \infty, f^{\prime}(x) \sim-x^{-2}, f^{\prime \prime}(x) \sim 2 x^{-3}$, and $f^{\prime \prime \prime}(x) \sim-6 x^{-4}$. Hence, if (4.27) holds for all large $A$ (or just a sequence $A \rightarrow \infty$ ), we obtain by taking the limit

$$
\frac{y f^{\prime \prime}(y)}{f^{\prime}(y)}=\lim _{x \rightarrow \infty} \frac{x f^{\prime \prime}(x)}{f^{\prime}(x)}=-2 \quad \text { and } \quad \frac{y^{2} f^{\prime \prime \prime}(y)}{f^{\prime}(y)}=\lim _{x \rightarrow \infty} \frac{x^{2} f^{\prime \prime \prime}(x)}{f^{\prime}(x)}=6 .
$$


Finally, let $F(x):=x f(x)=1-(1+x) e^{-x}$. Then $F^{\prime \prime}(y)=y f^{\prime \prime}(y)+2 f^{\prime}(y)=0$ and $F^{\prime \prime \prime}(y)=$ $y f^{\prime \prime \prime}(y)+3 f^{\prime \prime}(y)=6 y^{-1} f^{\prime}(y)-6 y^{-1} f^{\prime}(y)=0$. On the other hand, $F^{\prime}(x)=x e^{-x}, F^{\prime \prime}(x)=$ $(1-x) e^{-x}, F^{\prime \prime \prime}(x)=(x-2) e^{-x}$, so there is no solution to $F^{\prime \prime}(y)=y F^{\prime \prime \prime}(y)=0$. This contradiction finally proves that $\varphi^{\prime \prime \prime}(\tilde{p}) \neq 0$, at least for large $A$.

\section{Bootstrap percolation in random regular graphs}

Bootstrap percolation on a graph $G$ is a process that can be regarded as a model for the spread of an infection. We start by infecting a subset $\mathscr{A}_{0}$ of the vertices; typically we let $\mathscr{A}_{0}$ be a random subset of the vertex set $V(G)$ such that each vertex is infected with some given probability $q$, independently of all other vertices, but other choices are possible, including a deterministic choice of $\mathscr{A}_{0}$. Then, for some given threshold $\ell \in \mathbb{N}$, every uninfected vertex that has at least $\ell$ infected neighbours becomes infected. (Infected vertices stay infected; they never recover.) This is repeated until there are no further infections. We let $\mathscr{A}_{f}=\mathscr{A}_{f}^{(\ell)}$ be the final set of infected vertices. (This is perhaps not a good model for infectious diseases, but may be reasonable as a model for the spread of rumors or beliefs: you are skeptical the first time you hear something but get convinced the $\ell$ th time.)

Bootstrap percolation is more or less the opposite to taking the $k$-core. For regular graphs, there is an exact correspondence: it is easily seen that if the common vertex degree in $G$ is $d$, then the set $V(G) \backslash \mathscr{A}_{f}^{(\ell)}$ of finally uninfected vertices equals the $(d+1-\ell)$-core of the set $V(G) \backslash$ $\mathscr{A}_{0}$ of initially uninfected vertices. Furthermore, if the initial infection is random, with vertices infected independently with a common probability $q$, then the initial infection can be seen as a site percolation, where each vertex remains uninfected with probability $\pi=1-q$. Consequently, in this case we obtain results on the size of the final uninfected set from Theorem 4.5, taking $k=d-\ell+1$ and $\pi=1-q$.

Bootstrap percolation on the random regular graph $G(n, d)$ with fixed vertex degree $d$ was studied by Balogh and Pittel [1]. We can recover a large part of their results from Theorem 4.5. We have, as just said, $k=d-\ell+1$ and $\pi=1-q$. Moreover, all degrees $d_{i}^{(n)}=d$; hence the definitions in Section 2 yield $n_{j}=n \delta_{j d}, p_{j}=\delta_{j d}, \hat{D}_{n}=d, D=d$ and $\lambda=\mathbb{E} D=d$. Condition 2.1 is satisfied trivially. Furthermore, (4.2) and (4.3) yield, since $D_{p} \sim \operatorname{Bi}(d, p)$,

$$
\begin{aligned}
h(p) & =\sum_{j=k}^{d} j b_{d j}(p)=\sum_{j=k}^{d} j\left(\begin{array}{l}
d \\
j
\end{array}\right) p^{j}(1-p)^{d-j}=\sum_{j=k}^{d} d p\left(\begin{array}{c}
d-1 \\
j-1
\end{array}\right) p^{j-1}(1-p)^{d-j} \\
& =d p \mathbb{P}(\operatorname{Bi}(d-1, p) \geq k-1)=d p \mathbb{P}(\operatorname{Bi}(d-1, p) \geq d-\ell) \\
& =d p \mathbb{P}(\operatorname{Bi}(d-1,1-p) \leq \ell-1)
\end{aligned}
$$

and

$$
h_{1}(p)=\mathbb{P}(\operatorname{Bi}(d, p) \geq k)=\mathbb{P}(\operatorname{Bi}(d, p) \geq d-\ell+1)=\mathbb{P}(\operatorname{Bi}(d, 1-p) \leq \ell-1) .
$$

Consequently, (4.9) yields

$$
\pi_{c}:=\inf _{0<p \leq 1} \frac{d p^{2}}{h(p)}=\inf _{0<p \leq 1} \frac{p}{\mathbb{P}(\operatorname{Bi}(d-1,1-p) \leq \ell-1)} .
$$


We define $q_{c}:=1-\pi_{c}$, and Theorem 4.5 translates as follows. (Recall that we have proven Theorem 4.5 for the random multigraph $G^{*}(n, d)$, but as said in the introduction, the result holds for the simple random graph $G(n, d)$ by a standard conditioning.)

Theorem 5.1 ([1]). Let $d, \ell$ and $q \in[0,1]$ be given with $1 \leq \ell \leq d-1$. Consider bootstrap percolation on the random d-regular graph $G(n, d)$, with threshold $\ell$ and vertices initially infected randomly with probability $q$, independently of each other. Let

$$
q_{\mathrm{c}}=q_{\mathrm{c}}^{(\ell)}:=1-\inf _{0<p \leq 1} \frac{p}{\mathbb{P}(\operatorname{Bi}(d-1,1-p) \leq \ell-1)} .
$$

(i) If $q>q_{c}$, then $\left|\mathscr{A}_{f}\right|=n-o_{p}(n)$. Furthermore, if $l \leq d-2$ then w.h.p. $\left|\mathscr{A}_{f}\right|=n$, i.e., all vertices eventually become infected.

(ii) If $q<q_{c}$, then w.h.p. a positive proportion of the vertices remain uninfected, More precisely, if $\widehat{p}=\widehat{p}(q)$ is the largest $p \leq 1$ such that $\mathbb{P}(\operatorname{Bi}(d-1,1-p) \leq \ell-1) / p=(1-q)^{-1}$, then

$$
\left|\mathscr{A}_{f}\right| / n \stackrel{\mathrm{p}}{\longrightarrow} 1-(1-q) \mathbb{P}(\operatorname{Bi}(d, 1-\widehat{p}) \leq \ell-1)<1 .
$$

Proof. It remains only to show that in case (ii), $\widehat{p}$ is not a local maximum point of $\bar{\varphi}(p):=$ $h(p) /\left(d p^{2}\right)=\mathbb{P}(\operatorname{Bi}(d-1,1-p) \leq \ell-1) / p$. (In the notation of Section 4, $\bar{\varphi}(p)=\varphi(p) / \lambda$.) In fact, some simple calculus shows, see $\left[1, \S 3.2\right.$, where $\left.R(y)=\bar{\varphi}(y)^{-1}\right]$ for details, that the function $\bar{\varphi}$ is unimodal when $\ell<d-1$ and decreasing when $\ell=d-1$; thus there is no local maximum point when $\ell=d-1$, and otherwise the only local maximum point is the global maximum point $p_{0}$ with $\bar{\varphi}\left(p_{0}\right)=\pi_{\mathrm{c}}^{-1}=\left(1-q_{\mathrm{c}}\right)^{-1}$. (It follows also [1] that the equation $\bar{\varphi}(p)=(1-q)^{-1}$ in (ii) has exactly two roots for every $q<q_{c}$ when $\ell<d-1$ and one when $\ell=d-1$.)

Remark 5.2. The case $\ell=1(k=d)$ is rather trivial; in this case, $\mathscr{A}_{f}$ is the union of all components of $G(n, d)$ that contain at least one initially infected vertex. If further $d \geq 3$, then $G(n, d)$ is w.h.p. connected, and thus any non-empty $\mathscr{A}_{0}$ w.h.p. yields $\left|\mathscr{A}_{f}\right|=n$. (The case $d=2$ is different but also simple: $G(n, 2)$ consists of disjoint cycles, and only a few small cycles will remain uninfected.)

Actually, Balogh and Pittel [1] study primarily the case when the initially infected set $\mathscr{A}_{0}$ is deterministic; they then derive the result above for a random $\mathscr{A}_{0}$ by conditioning on $\mathscr{A}_{0}$. Thus, assume now that $\mathscr{A}_{0}$ is given, with $\left|\mathscr{A}_{0}\right|=m$. (For $G(n, d)$, because of the symmetry, it does not matter whether we remove a specified set of $m$ vertices or a uniformly distributed random set with $m$ vertices.) Assuming $m \sim n q$, we have the same results in this case, see Remark 1.1; indeed, the proof is slightly simpler since the use of the law of large numbers in Subsection 2.1 is replaced by the obvious $\tilde{n}_{d}=n-m, \tilde{n}_{1}=n_{+}=d m$.

Theorem 5.3. Theorem 5.1 remains valid if the initially infected set is any given set with $m=m(n)$ vertices, where $m / n \rightarrow q$.

Remark 5.4. As in Remark 4.8, it is also possible to study the threshold in greater detail by allowing $q$ to depend on $n$. If we assume $\ell \leq d-2$ (i.e., $k \geq 3$ ) and $q=q(n) \rightarrow q_{\mathrm{c}}$ defined by (5.1), then Janson and Luczak [11], Theorem 3.5 applies and implies the following, also proved by Balogh and Pittel [1] by different methods. 
Theorem 5.5 ([1]). Consider bootstrap percolation on $G(n, d)$ with $\ell \leq d-2$, and assume that the set $\mathscr{A}_{0}$ of initially infected vertices either is deterministic with $\left|\mathscr{A}_{0}\right|=q(n) n$ or random, with each vertex infected with probability $q(n)$.

(i) If $q(n)-q_{c} \gg n^{-1 / 2}$, then w.h.p. $\left|\mathscr{A}_{f}\right|=n$, i.e., all vertices become infected.

(ii) If $q_{\mathrm{c}}-q(n) \gg n^{-1 / 2}$, then w.h.p. $\left|\mathscr{A}_{f}\right|<n$ and, moreover,

$$
\left|\mathscr{A}_{f}\right|=h_{1}(\widehat{p}(q(n))) n+O_{p}\left(n^{1 / 2}\left|q(n)-q_{\mathrm{c}}\right|^{-1 / 2}\right) .
$$

Janson and Luczak [11], Theorem 3.5 is stated for random multigraphs, and for $G^{*}(n, d)$ it yields further an asymptotic normal distribution in case (ii), as well as a precise result for $\mathbb{P}\left(\left|\mathscr{A}_{f}\right|=n\right)$ in the transition window $q(n)-q_{c}=O\left(n^{-1 / 2}\right)$. The latter result can easily be transformed into the following analogue of [11, Theorem 1.4]; the asymptotic variance $\sigma^{2}$ is given by explicit but rather complicated formulas in [11].

Theorem 5.6. Assume $\ell \leq d-2$. Infect (from the outside) the vertices in $G^{*}(n, d)$ one by one in random order, letting the infection spread as above to every vertex having at least $\ell$ infected neighbours, and let $M$ be the number of externally infected vertices required to make $\left|\mathscr{A}_{f}\right|=n$. Then $\left(M-n q_{\mathrm{c}}\right) / n^{1 / 2} \stackrel{\mathrm{d}}{\longrightarrow}$ $N\left(0, \sigma^{2}\right)$, with $\sigma^{2}>0$.

Presumably, the same results hold for $G(n, d)$, but technical difficulties have so far prevented a proof, cf. [11]. In any case, it follows from Theorem 5.6 that the size of the transition window is $O\left(n^{-1 / 2}\right)$ for $G(n, d)$ too, and not smaller.

\section{A The $k$-core and branching processes}

We give a precise statement of the relation between Theorem 4.1 and branching processes. This can be seen heuristically from the branching process approximation of the local exploration process, but as said above, we do not attempt to make this approximation rigorous; instead we compare the quantities in Theorem 4.1 with branching process probabilities.

Theorem A.1. Let $\mathscr{X}$ be a Galton-Watson branching process with offspring distribution $D^{*}$ and starting with one individual $o$, and let $\overline{\mathscr{X}}$ be the modified branching process where the root o has offspring distribution $D$ but everyone else has offspring distribution $D^{*}$. We regard these branching processes as (possibly infinite) trees with root $o$. Further, let $\mathscr{T}_{k}$ be the infinite rooted tree where each node has $k-1$ children, and let $\overline{\mathscr{T}}_{k}$ be the infinite rooted $k$-regular tree where the root has $k$ children and everyone else $k-1$.

Then $\widehat{p}=\mathbb{P}\left(\mathscr{X} \supseteq \mathscr{T}_{k}\right)$, the probability that $\mathscr{X}$ contains a rooted copy of $\mathscr{T}_{k}$ (i.e., a copy of $\mathscr{T}_{k}$ with root o) and $h_{1}(\widehat{p})=\mathbb{P}\left(\overline{\mathscr{X}} \supseteq \overline{\mathscr{T}}_{k}\right)$, the probability that $\overline{\mathscr{X}}$ contains a rooted copy of $\overline{\mathscr{T}}_{k}$.

Hence, by Theorem 4.1, the probability that a random vertex belongs to the $k$-core, which is $\mathbb{E}\left(v\left(\right.\right.$ Core $\left.\left._{k}^{*}\right) / n\right)$, converges to the probability $\mathbb{P}\left(\overline{\mathscr{X}} \supseteq \overline{\mathscr{T}}_{k}\right)$, the probability that the branching process approximating the local structure at a random vertex contains the infinite $k$-regular tree $\overline{\mathscr{T}}_{k}$. Similarly, the probability that a random edge belongs to the $\operatorname{Core}_{k}^{*}$, which is $\sim \mathbb{E}\left(e\left(\operatorname{Core}_{k}^{*}\right) /(n \lambda / 2)\right)$, converges to $h(\widehat{p}) / \lambda=\widehat{p}^{2}=\mathbb{P}\left(\mathscr{X} \supseteq \mathscr{T}_{k}\right)^{2}$, which can be interpreted as the probability that both endpoints of a random edge grow infinite $k$-regular trees in the branching process approximation. 
Proof. Let $\mathscr{T}_{k n}$ be the subtree of $\mathscr{T}_{k}$ consisting of all nodes of height $\leq n$, i.e., the rooted tree of height $n$ where each node of height $<n$ has $k-1$ children, and let $q_{n}$ be the probability that $\mathscr{X}$ contains a copy of $\mathscr{T}_{k n}$. Thus, $q_{0}=1$, and for $n \geq 0, q_{n+1}$ is the probability that the root $o$ has at least $k-1$ children that each is the root of a copy of $\mathscr{T}_{k n}$ in the corresponding subtree of $\mathscr{X}$; let us call such children good. By the branching property, the subtrees rooted at the children of $o$ are independent copies of $\mathscr{X}$ : thus the probability that a given child is good is $q_{n}$, and the number of good children of $o$ has the tinned distribution $D_{q_{n}}^{*}$. Hence,

$$
\begin{aligned}
q_{n+1} & =\mathbb{P}\left(D_{q_{n}}^{*} \geq k-1\right)=\sum_{d=k}^{\infty} \mathbb{P}\left(D^{*}=d-1\right) \sum_{l=k}^{\infty} \mathbb{P}\left(\operatorname{Bi}\left(d-1, q_{n}\right)=l-1\right) \\
& =\sum_{d \geq k} \sum_{l \geq k} \frac{d}{\lambda} \mathbb{P}(D=d) \frac{l}{d q_{n}} \mathbb{P}\left(\operatorname{Bi}\left(d, q_{n}\right)=l\right) \\
& =\frac{1}{\lambda q_{n}} \sum_{l \geq k} l \mathbb{P}\left(D_{q_{n}}=l\right)=\frac{1}{\lambda q_{n}} h\left(q_{n}\right) .
\end{aligned}
$$

Since $x \mapsto h(x) /(\lambda x)$ is increasing (e.g. by the same calculation) and $1=q_{0} \geq q_{1} \geq \ldots$, it follows that $q_{n}$ decreases to the largest root $\widehat{p}$ of $\frac{1}{\lambda q} h(q)=q$ in $[0,1]$. On the other hand, the events $\mathscr{E}_{n}:=\left\{\mathscr{X} \supseteq \mathscr{T}_{k n}\right\}$ are decreasing, $\mathscr{E}_{1} \supseteq \mathscr{E}_{2} \supseteq \cdots$, and $\bigcap_{n} \mathscr{E}_{n}$ is, by a compactness argument, equal to the event $\left\{\mathscr{X} \supseteq \mathscr{T}_{k}\right\}$. Hence, $\mathbb{P}\left(\mathscr{X} \supseteq \mathscr{T}_{k}\right)=\lim _{n} q_{n}=\widehat{p}$.

Similarly, $\overline{\mathscr{X}}$ contains a rooted copy of $\overline{\mathscr{T}}_{k}$ if and only if the root $o$ has at least $k$ good (now with $n=\infty$ ) children. We have shown that each child is good with probability $\widehat{p}$, and thus the number of good children has the thinned distribution $D_{\widehat{p}}$; hence $\mathbb{P}\left(\overline{\mathscr{X}} \supseteq \overline{\mathscr{T}}_{k}\right)=\mathbb{P}\left(D_{\widehat{p}} \geq k\right)=h_{1}(\widehat{p})$.

Remark A.2. When $k=2, \mathscr{T}_{2}$ is just an infinite path, and thus $\widehat{p}=\mathbb{P}\left(\mathscr{X} \supseteq \mathscr{T}_{2}\right)$ is just the survival probability $\rho$ of the branching process $\mathscr{X}$, as observed algebraically in Remark 4.4. Hence the thresholds for 2-core and giant component coincide, for any of our percolation models. Moreover, we see that if $v$ is a random vertex, the events " $v$ is in a giant component" and " $v$ is in the 2-core" are approximated by "the root $o$ in $\overline{\mathscr{X}}$ has at least one child with infinite progeny" and "the root $o$ in $\overline{\mathscr{X}}$ has at least two children with infinite progeny", respectively, which again shows the close connection between these properties.

Acknowledgement. Part of this research was done during visits to the University of Cambridge and Trinity College in 2007, the 11th Brazilian School of Probability in Maresias, August 2007, and the Isaac Newton Institute and Churchill College in Cambridge in 2008. I thank Louigi Addario-Berry, Malwina Luczak, Rob Morris and James Norris for stimulating discussions and helpful comments.

\section{References}

[1] J. Balogh, B. G. Pittel, Bootstrap percolation on the random regular graph. Random Structures Algorithms 30 (2007), no. 1-2, 257-286. MR2283230

[2] B. Bollobás, The evolution of sparse graphs. Graph theory and Combinatorics (Cambridge, 1983), 35-57, Academic Press, London, 1984. MR0777163

[3] B. Bollobás, Random Graphs, 2nd ed., Cambridge Univ. Press, Cambridge, 2001. MR1864966 
[4] T. Britton, S. Janson \& A. Martin-Löf, Graphs with specified degree distributions, simple epidemics and local vaccination strategies. Advances Appl. Probab. 39 (2007), no. 4, 922-948. MR2381582

[5] J. Cain \& N. Wormald, Encore on cores. Electronic J. Combinatorics, 13 (2006), no. 1, R81. MR2255423

[6] R. W. R. Darling, D. A. Levin \& J. R. Norris, Continuous and discontinuous phase transitions in hypergraph processes. Random Structures Algorithms 24 (2004), no. 4, 397-419. MR2060628

[7] N. Fountoulakis, Percolation on sparse random graphs with given degree sequence. Preprint, 2007. arXiv:math/0703269v1.

[8] A. Gut, Probability: A Graduate Course. Springer, New York, 2005. MR2125120

[9] S. Janson, The probability that a random multigraph is simple. Combin. Probab. Comput., to appear. arXiv:math. CO/0609802.

[10] S. Janson \& M. J. Luczak, A simple solution to the k-core problem. Random Structures Algorithms 30 (2007), 50-62. MR2283221

[11] S. Janson \& M. J. Luczak, Asymptotic normality of the $k$-core in random graphs. Ann. Appl. Probab., 18 (2008), no. 3, 1085-1137. MR2418239

[12] S. Janson \& M. J. Luczak, A new approach to the giant component problem. Random Structures Algorithms, to appear. arXiv:0707.1786v1.

[13] O. Kallenberg, Foundations of Modern Probability, 2nd ed., Springer-Verlag, New York, 2002. MR1876169

[14] T. Łuczak, Size and connectivity of the k-core of a random graph, Discr. Math. 91 (1991) 61-68. MR1120887

[15] M. Molloy \& B. Reed, A critical point for random graphs with a given degree sequence, Random Structures Algorithms 6 (1995), no. 2-3, 161-179. MR1370952

[16] M. Molloy \& B. Reed, The size of the giant component of a random graph with a given degree sequence, Combin. Probab. Comput. 7 (1998), no. 3, 295-305. MR1664335

[17] B. Pittel, J. Spencer \& N. Wormald, Sudden emergence of a giant $k$-core in a random graph, $J$. Combin. Theor. Ser. B 67 (1996), 111-151. MR1385386

[18] O. Riordan, The $k$-core and branching processes. Combin. Probab. Comput. 17 (2008), no. 1, 111-136. MR2376426 